\title{
Exact relations for twist-3 gluon distribution and fragmentation functions from operator identities
}

\author{
Yuji Koike $\odot{ }^{2}$ Kenta Yabe $\odot,^{3}$ and Shinsuke Yoshida $\odot^{1}$ \\ ${ }^{1}$ Institute of Quantum Matter and School of Physics and Telecommunication Engineering, \\ South China Normal University, Guangzhou 510006, China \\ ${ }^{2}$ Department of Physics, Niigata University, Ikarashi, Niigata 950-2181, Japan \\ ${ }^{3}$ Graduate School of Science and Technology, Niigata University, Ikarashi, Niigata 950-2181, Japan
}

(Received 29 December 2019; accepted 24 February 2020; published 12 March 2020)

\begin{abstract}
We perform a systematic study on the twist-3 gluon distribution and fragmentation functions which appear in the collinear twist-3 factorization for hard inclusive processes. Three types of twist-3 distribution and fragmentation functions, i.e., intrinsic, kinematical, and dynamical ones, which are necessary to describe all kinds of twist- 3 cross sections, are related to each other by the operator identities based on the QCD equation of motion and the Lorentz invariance properties of the correlation functions. We derive the exact relations for all twist- 3 gluonic distribution and fragmentation functions for a spin- $1 / 2$ hadron. Those relations allow one to express intrinsic and kinematical twist-3 gluon functions in terms of the twist-2 and dynamical twist-3 functions, which provides a basis for the renormalization of intrinsic and kinematical twist-3 functions. In addition, those model independent relations are crucial to guarantee gauge invariance and frame independence properties of the twist- 3 cross sections.
\end{abstract}

DOI: 10.1103/PhysRevD.101.054017

\section{INTRODUCTION}

During the past few decades twist-3 effects in (semi-) inclusive processes have been receiving great attention, in that they show up as a leading contribution to interesting spin asymmetries and reveal an important role of multiparton correlations in the scattering processes which shed new lights on the hadron structure. By now theoretical methods for dealing with those twist-3 effects have been developed and widely used to derive many relevant twist-3 cross section formulas. Such theoretical studies include those for $g_{2}$-structure function of the nucleon measured in deep-inelastic scattering [1,2], single spin asymmetries (SSA) for a hadron or (virtual) photon production in proton-proton (nucleus) collisions with one proton transversely polarized, $p^{\uparrow} p \rightarrow h X\left(h=\pi, D, \gamma, \gamma^{*}\right.$ etc.) [3-17], $p^{\uparrow} A \rightarrow h X$ [18-22], and semi-inclusive deep-inelastic scattering (SIDIS), $e p^{\uparrow} \rightarrow e h X$ [23-32], SSA in transversely polarized hyperon production in the unpolarized proton-proton collision, $p p \rightarrow \Lambda^{\uparrow} X$ [33-38] and in $e^{+} e^{-}$ -collision, $e^{+} e^{-} \rightarrow \Lambda^{\uparrow} X$ [39], the longitudinal-transverse double spin asymmetry $A_{\mathrm{LT}}$ in the proton-proton collision,

Published by the American Physical Society under the terms of the Creative Commons Attribution 4.0 International license. Further distribution of this work must maintain attribution to the author(s) and the published article's title, journal citation, and DOI. Funded by SCOAP ${ }^{3}$. $\vec{p} p^{\uparrow} \rightarrow\left\{h, \gamma^{*}\right\} X$ [40-44], etc. Collinear twist-3 parton distribution functions (DFs) and fragmentation functions (FFs) which appear in these twist-3 factorization formula for the cross sections no longer have probability interpretation but represent multiparton (quark-gluon or purely gluonic) correlations in the hadrons or in the fragmentation processes. The leading order evolution equations for the relevant twist-3 functions have been also derived $[10,30,45-58]$.

Collinear twist-3 DFs and FFs can be, in general, classified into three types: intrinsic, kinematical, and dynamical ones [59]. Although they all appear in the calculation of the twist-3 cross section formula, they are not independent from each other, but are related by QCD equation of motion (e.o.m.) and Lorentz invariance properties of the correlation functions. One of the present authors (Y. K.) performed a systematic study on the twist-3 quark DFs and FFs, and presented a complete set of those model independent relations, which are often called e.o.m. relations and the Lorentz invariance relations [59]. These relations allow one to express the intrinsic and kinematical twist-3 DFs and FFs in terms of the twist-2 functions and the dynamical twist-3 functions. In addition, they play a critical role to guarantee the gauge invariance and frame independence of the twist- 3 cross sections $[29,59,60]$. In this paper, we extend the study to gluonic twist-3 DFs and FFs for a spin- $1 / 2$ hadron $[37-39,61,62]$ and derive all of those exact relations. For the twist-3 gluon DFs in the transversely polarized nucleon, which are relevant to SSAs 
in $e p^{\uparrow} \rightarrow e D X$ [27], $p^{\uparrow} p \rightarrow D X$ [11], $p^{\uparrow} p \rightarrow\left\{\gamma, \gamma^{*}\right\} X$ [12], and also $A_{\mathrm{LT}}$ for $\vec{p} p^{\uparrow} \rightarrow D X$ [42], one of the present authors (S. Y.) derived such relations [63], while no such systematic studies exist for the twist-3 gluon FFs. There are several purely gluonic twist-3 FFs for a transversely polarized spin- $1 / 2$ hadron, so the present study is particularly important for the study of their contribution to the polarized hyperon production in the unpolarized proton-proton collision $\left(p p \rightarrow \Lambda^{\uparrow} X\right)$ [37,38] and SIDIS $\left(e p \rightarrow e \Lambda^{\uparrow} X\right.$ ), etc. Those exact relations for the gluonic DFs and FFs need to be taken into account in the derivation of the cross section and will be crucial to guarantee gauge invariance and the frame independence of the twist- 3 cross sections as in the case of quark DFs and FFs.

The remainder of this paper is organized as follows: In Sec. II, we derive the relations among the twist-3 gluon DFs. After summarizing the complete set of purely gluonic distributions up to twist-3, we derive all the constraint relations among those functions. In Sec. III, we extend the study to the twist- 3 gluon FFs. There are more twist-3 FFs compared to the twist-3 DFs due to the lack of a constraint from time reversal invariance. In particular, the dynamical FFs become complex, and the real and imaginary parts obey different constraint relations. Section IV will be devoted to a brief summary.

\section{TWIST-3 GLUON DISTRIBUTIONS}

\section{A. Intrinsic, kinematical, and dynamical twist-3 gluon distributions}

We first summarize the definition of three types of purely gluonic distribution functions in the nucleon which has mass $M$, momentum $P\left(P^{2}=M^{2}\right)$, and the spin vector $S$ $\left(S^{2}=-M^{2}\right)$. As usual, we introduce two lightlike vectors $p$ and $n$, which satisfy $P^{\mu}=p^{\mu}+\frac{M^{2}}{2} n^{\mu}$ and $p \cdot n=1$. Below we work in a frame where $p^{\mu}=P^{+} g_{+}^{\mu}$ and $n^{\mu}=\left(1 / p^{+}\right) g_{-}^{\mu}$. The simplest collinear gluon distribution functions are defined from the light cone correlation functions of the gluon's field strengths $F_{a}^{\alpha \nu}$ with color index $a$ in the nucleon state $|P S\rangle$ as $[61,62]$

$$
\begin{aligned}
\Phi^{\alpha \nu, \beta \mu}(x)= & \int \frac{d \lambda}{2 \pi} e^{i \lambda x}\left\langle P S\left|F_{a}^{\alpha \nu}(0)[0, \lambda n]_{a b} F_{b}^{\beta \mu}(\lambda n)\right| P S\right\rangle \\
= & \frac{1}{2}\left\{\left(-g_{\perp}^{\alpha \beta} p^{\mu}+g_{\perp}^{\alpha \mu} p^{\beta}\right) p^{\nu}-\left(-g_{\perp}^{\nu \beta} p^{\mu}+g_{\perp}^{\nu \mu} p^{\beta}\right) p^{\alpha}\right\} x G(x) \\
& +\frac{i}{2}(S \cdot n)\left\{\left(\epsilon^{p n \alpha \beta} p^{\mu}-\epsilon^{p n \alpha \mu} p^{\beta}\right) p^{\nu}-\left(\epsilon^{p n \nu \beta} p^{\mu}-\epsilon^{p n \nu \mu} p^{\beta}\right) p^{\alpha}\right\} x \Delta G(x) \\
& -\frac{i M}{2}\left\{\left(\epsilon^{\alpha \beta n S_{\perp}} p^{\mu}-\epsilon^{\alpha \mu n S_{\perp}} p^{\beta}\right) p^{\nu}-\left(\epsilon^{\nu \beta n S_{\perp}} p^{\mu}-\epsilon^{\nu \mu n S_{\perp}} p^{\beta}\right) p^{\alpha}\right\} x \Delta G_{3 T}(x) \\
& +\frac{i M}{2}\left\{\epsilon^{\beta \mu p n}\left(S_{\perp}^{\alpha} p^{\nu}-S_{\perp}^{\nu} p^{\alpha}\right)-\epsilon^{\alpha \nu p n}\left(S_{\perp}^{\beta} p^{\mu}-S_{\perp}^{\mu} p^{\beta}\right)\right\} x \Delta H_{3 T}(x)+\cdots,
\end{aligned}
$$

where $+\cdots$ denotes twist-4 or higher, $g_{\perp}^{\alpha \beta}=$ $g^{\alpha \beta}-p^{\alpha} n^{\beta}-p^{\beta} n^{\alpha}$, and the transverse spin vector $S_{\perp}^{\mu}$ is defined as $S^{\mu}=(S \cdot n) p^{\mu}+(S \cdot p) n^{\mu}+M S_{\perp}^{\mu} \cdot[0, \lambda n] \equiv$ $P \exp \left\{i g \int_{\lambda}^{0} d t A(t n) \cdot n\right\}$ is the gauge link which guarantees gauge invariance of the correlation function. Here and below we use the shorthand notation $\epsilon^{p n \alpha \beta} \equiv \epsilon^{\nu \mu \alpha \beta} p_{\nu} n_{\mu}$, etc. $G(x)$ and $\Delta G(x)$ are, respectively, twist-2 unpolarized and helicity distributions and $\Delta G_{3 T}(x)$ and $\Delta H_{3 T}(x)$ are the intrinsic twist-3 distributions corresponding, respectively, to $\left\langle F^{+\perp} F^{+-}\right\rangle$and $\left\langle F^{+\perp} F^{\perp \perp}\right\rangle$ correlators. Although $\Delta H_{3 T}(x)$ drops from the correlator $\Phi^{\alpha n, \beta n}(x)$ which contributes to a cross section, we need the form (1) to derive constraint relations among the twist-3 distributions. Each function in (1) has a support on $|x|<1$.

The second type of the twist -3 gluon distributions are the kinematical ones which are defined as

$$
\begin{aligned}
\Phi_{\partial}^{\alpha \beta \gamma}(x)= & \int \frac{d \lambda}{2 \pi} e^{i \lambda x}\left\langle P S\left|F^{\alpha n}(0) F^{\beta n}(\lambda n)\right| P S\right\rangle i \overleftarrow{\partial}^{\gamma} \\
\equiv & \lim _{\xi \rightarrow 0} \int \frac{d \lambda}{2 \pi} e^{i \lambda x}\left\langle P S\left|\left(F^{\alpha n}(0)[0, \infty n]\right)_{a} i \frac{d}{d \xi_{\gamma}}\left([\infty n+\xi, \lambda n+\xi] F^{\beta n}(\lambda n+\xi)\right)_{a}\right| P S\right\rangle \\
= & \frac{M}{2} g_{\perp}^{\alpha \beta} \epsilon^{p n S_{\perp \gamma}} G_{T}^{(1)}(x)-\frac{i M}{2} \epsilon^{p n \alpha \beta} S_{\perp}^{\gamma} \Delta G_{T}^{(1)}(x)+\frac{M}{8}\left(\epsilon^{p n S_{\perp}\{\alpha} g_{\perp}^{\beta\} \gamma}+\epsilon^{p n \gamma\{\alpha} S_{\perp}^{\beta\}}\right) \Delta H_{T}^{(1)}(x) \\
& +\left(\text { terms proportional to } p^{\gamma}\right)+\cdots,
\end{aligned}
$$


where $\cdots$ denotes twist- 4 or higher. These three kinematical distributions $\Delta G_{T}^{(1)}(x), \Delta G_{T}^{(1)}(x)$, and $\Delta H_{T}^{(1)}(x)$ can be also written as the $k_{\perp}^{2} / M^{2}$-moment of the transverse momentum dependent (TMD) distributions. Note that the TMD distribution corresponding to $\Delta G_{T}^{(1)}(x)$ is naively $T$ - even, while those for $G_{T}^{(1)}(x)$ and $\Delta H_{T}^{(1)}(x)$ are naively $T$-odd. Each function in (2) has a support on $|x|<1$.

The last distributions (third type) are the dynamical ones which are defined as the light cone correlation functions of three field strengths ("F-type" distribution) $[27,61]^{1}$ :

$$
\begin{aligned}
N_{F}^{\alpha \beta \gamma}\left(x_{1}, x_{2}\right) & =i \int \frac{d \lambda}{2 \pi} \int \frac{d \mu}{2 \pi} e^{i \lambda x_{1}} e^{i \mu\left(x_{2}-x_{1}\right)}\left\langle P S\left|i f^{a c b}\left(F^{n \alpha}(0)[0, \mu n]\right)_{a} g F_{c}^{n \gamma}(\mu n)\left([\mu n, \lambda n] F^{n \beta}(\lambda n)\right)_{b}\right| P S\right\rangle \\
& =2 i M\left[-g_{\perp}^{\alpha \beta} \epsilon^{\gamma p n S_{\perp}} N\left(x_{1}, x_{2}\right)+g_{\perp}^{\alpha \gamma} \epsilon^{\beta p n S_{\perp}} N\left(x_{2}, x_{2}-x_{1}\right)+g_{\perp}^{\beta \gamma} \epsilon^{\alpha p n S_{\perp}} N\left(x_{1}, x_{1}-x_{2}\right)\right]+\cdots
\end{aligned}
$$

where $f^{a c b}$ is the antisymmetric structure constant for color $\mathrm{SU}(\mathrm{N})$ and $\cdots$ denotes twist- 4 or higher. $N\left(x_{1}, x_{2}\right)$ satisfies the symmetry relation $N\left(x_{1}, x_{2}\right)=N\left(x_{2}, x_{1}\right)$ and $N\left(-x_{1},-x_{2}\right)=-N\left(x_{1}, x_{2}\right)$ and has a support on $\left|x_{1,2}\right|<1$ and $\left|x_{1}-x_{2}\right|<1$. Replacing if $f^{a b c}$ by $d^{a b c}$ (symmetric structure constants) in $N_{F}^{\alpha \beta \gamma}\left(x_{1}, x_{2}\right)$, one can define other 3-gluon correlation functions. However, we shall not consider them, since they are not related to any other types of twist-3 gluon distributions. We call $N\left(x_{1}, x_{2}\right)$ [and $G_{F}\left(x_{1}, x_{2}\right)$ in (17) below] dynamical twist-3 DFs. Replacing $g F^{2 \gamma}(\mu n)$ by the covariant derivative $D^{\gamma}(\mu n)=$ $\partial^{\gamma}-i g A^{\gamma}(\mu n)$ in (3), one obtains " $D$-type" distributions as

$$
\begin{aligned}
N_{D}^{\alpha \beta \gamma}\left(x_{1}, x_{2}\right)= & i \int \frac{d \lambda}{2 \pi} \int \frac{d \mu}{2 \pi} e^{i \lambda x_{1}} e^{i \mu\left(x_{2}-x_{1}\right)}\left\langle P S\left|\left(F^{n \alpha}(0)[0, \mu n]\right)_{a}\left(D^{\gamma}(\mu n)[\mu n, \lambda n] F^{n \beta}(\lambda n)\right)_{a}\right| P S\right\rangle \\
= & 2 i M\left[-g_{\perp}^{\alpha \beta} \epsilon^{\gamma p n S_{\perp}} D_{1}\left(x_{1}, x_{2}\right)-g_{\perp}^{\alpha \gamma} \epsilon^{\beta p n S_{\perp}} D_{2}\left(x_{1}, x_{2}\right)+g_{\perp}^{\beta \gamma} \epsilon^{\alpha p n S_{\perp}} D_{2}\left(x_{2}, x_{1}\right)\right] \\
& -\frac{1}{2} g_{\perp}^{\alpha \beta} p^{\gamma} G\left(x_{1}, x_{2}\right) x_{2}^{2}+\frac{i}{2} \epsilon^{\alpha \beta p n} p^{\gamma}(S \cdot n) \Delta G\left(x_{1}, x_{2}\right) x_{2}^{2}-\frac{i}{2} M \epsilon^{\alpha \beta n S_{\perp}} p^{\gamma} \Delta G_{3 T}\left(x_{1}, x_{2}\right) x_{2}^{2}+\cdots
\end{aligned}
$$

where $\cdots$ denotes twist- 4 or higher. It is easy to see that $\int d x_{1} N_{D}^{\alpha \beta n}\left(x_{1}, x\right)$ is reduced to $\Phi^{\alpha n, \beta n}(x)$, and the three distributions in the last three terms of (4) are thus related to those in (1) as

$$
\int_{-1}^{1} d x_{1} f\left(x_{1}, x\right)=f(x), \quad \text { for } f=G, \Delta G, \Delta G_{3 T} .
$$

Equations (1), (2), (3) and (4) define all necessary collinear twist-3 gluonic distribution functions in the collinear twist3 formalism. Below we shall derive all constraint relations among those functions.

\section{B. Relations between $D$ - and $F$-type DFs and QCD equation of motion}

Using the identity

$$
\begin{aligned}
D^{\gamma}(\mu n)[\mu n, \lambda n]= & i g \int_{\lambda}^{\mu} d t[\mu n, t n] F^{\gamma n}(t n)[t n, \lambda n] \\
& +[\mu n, \lambda n] D^{\gamma}(\lambda n),
\end{aligned}
$$

$D$ - and $F$-type 3 -gluon correlators in (4) and (3) are connected as

\footnotetext{
${ }^{1}$ We follow the convention of [27].
}

$$
\begin{aligned}
N_{D}^{\alpha \beta \gamma}\left(x_{1}, x_{2}\right)= & \mathcal{P} \frac{1}{x_{2}-x_{1}} N_{F}^{\alpha \beta \gamma}\left(x_{1}, x_{2}\right) \\
& +\delta\left(x_{1}-x_{2}\right) \tilde{\Phi}_{\partial}^{\alpha \beta \gamma}\left(x_{1}\right)
\end{aligned}
$$

where

$$
\begin{aligned}
\tilde{\Phi}_{\partial}^{\alpha \beta \gamma}(x)= & i \int \frac{d \lambda}{2 \pi} e^{i \lambda x}\left\langle P S\left|F^{n \alpha}(0)[0, \lambda n] D^{\gamma}(\lambda n) F^{n \beta}(\lambda n)\right| P S\right\rangle \\
& +\int \frac{d \lambda}{2 \pi} e^{i \lambda x} \int_{-\infty}^{\infty} d \mu \frac{1}{2} \epsilon(\mu-\lambda)\langle P S| \\
& \times F^{n \alpha}(0) g F^{n \gamma}(\mu n) F^{n \beta}(\lambda n)|P S\rangle
\end{aligned}
$$

with $\epsilon(\mu-\lambda)=2 \theta(\mu-\lambda)-1$. Here and below we often suppress the color indices and gauge links for simplicity: $F^{n \alpha}(0)[0, \lambda n] D^{\gamma}(\lambda n) F^{n \beta}(\lambda n)$ denotes $\left(F^{n \alpha}(0)[0, \lambda n]\right)_{a} \times$ $\left(D^{\gamma}(\lambda n) F^{n \beta}(\lambda n)\right)_{a}$, and $F^{n \alpha}(0) g F^{n \gamma}(\mu n) F^{n \beta}(\lambda n)$ represents $i f^{a c b}\left(F^{n \alpha}(0)[0, \mu n]\right)_{a} g F_{c}^{n \gamma}(\mu n)\left([\mu n, \lambda n] F^{n \beta}(\lambda n)\right)_{b}$. On the other hand, the correlator for the kinematical twist-3 distributions $\Phi_{\partial}^{\alpha \beta \gamma}(x)$ in (2) can be rewritten as

$$
\begin{aligned}
\Phi_{\partial}^{\alpha \beta \gamma}(x)= & i \int \frac{d \lambda}{2 \pi} e^{i \lambda x}\left\langle P S\left|F^{n \alpha}(0)[0, \lambda n] D^{\gamma}(\lambda n) F^{n \beta}(\lambda n)\right| P S\right\rangle \\
& +\int \frac{d \lambda}{2 \pi} e^{i \lambda x} \int_{\lambda}^{\infty} d \mu\langle P S| \\
& \times F^{n \alpha}(0) g F^{n \gamma}(\mu n) F^{n \beta}(\lambda n)|P S\rangle
\end{aligned}
$$


One thus obtains the relation

$$
\tilde{\Phi}_{\partial}^{\alpha \beta \gamma}(x)=\Phi_{\partial}^{\alpha \beta \gamma}(x)+i \pi N_{F}^{\alpha \beta \gamma}(x, x),
$$

where $N_{F}^{\alpha \beta \gamma}(x, x)$ defines soft-gluon-pole functions. By comparing real and imaginary parts of both sides of (7), one obtains the following relations:

$$
\begin{aligned}
D_{1}\left(x_{1}, x_{2}\right)= & \mathcal{P} \frac{1}{x_{2}-x_{1}} N\left(x_{1}, x_{2}\right), \\
D_{2}\left(x_{1}, x_{2}\right)= & \mathcal{P} \frac{-1}{x_{2}-x_{1}} N\left(x_{2}, x_{2}-x_{1}\right) \\
& -\frac{1}{4} \delta\left(x_{1}-x_{2}\right) \Delta G_{T}^{(1)}\left(x_{1}\right), \\
G_{T}^{(1)}(x)= & 4 \pi(N(x, x)-N(x, 0)), \\
\Delta H_{T}^{(1)}(x)= & -8 \pi N(x, 0) .
\end{aligned}
$$

The first two relations were derived in [63]. They show the $D$-type functions are determined by the $F$-type and kinematical functions. The last two relations (13) and (14) are the analogs of the relations for the quark distributions that show the $k_{\perp}^{2}$-moment of the "naively $T$-odd" TMD distribution functions, such as Sivers and Boer-Mulders functions, and are proportional to the soft-gluon-pole (SGP) function of the $F$-type quark-gluon correlation function. It has been shown that the SGP functions $N(x, x)$ and $N(x, 0)$ contribute to SSAs for $e p^{\uparrow} \rightarrow e D X$ [27], $p^{\uparrow} p \rightarrow D X$ [11], $p^{\uparrow} p \rightarrow\left\{\gamma, \gamma^{*}\right\} X$ [12] and $p^{\uparrow} p \rightarrow \pi X$ [16]. To the best of our knowledge, the relations (13) and (14) were not explicitly written in the literature.

To get further relations, we multiply $g_{\beta \gamma}^{\perp}$ to (7), integrate over $x_{1}$ and use the relation $D_{\perp \beta}(\lambda n) F^{n \beta}(\lambda n)=$ $-D^{n}(\lambda n) F^{n p}(\lambda n)+g \bar{\psi}(\lambda n) h t^{a} \psi(\lambda n)$ which follows from the QCD equation of motion, $\left(D_{\mu} F^{\mu \alpha}\right)_{a}=-g \bar{\psi} \gamma^{\alpha} t^{a} \psi$. One then obtains

$$
\begin{aligned}
& \frac{x^{2}}{2} \Delta G_{3 T}(x)+D_{g}(x) \\
& =2 \int_{-1}^{1} d x_{1} \mathcal{P} \frac{1}{x-x_{1}}\left[-N\left(x_{1}, x\right)+N\left(x, x-x_{1}\right)\right. \\
& \left.\quad+2 N\left(x_{1}, x_{1}-x\right)\right]-\frac{1}{2} \Delta G_{T}^{(1)}(x),
\end{aligned}
$$

where $D_{g}(x)$ is defined as

$M D_{g}(x) \epsilon^{\alpha p n S_{\perp}}=\int \frac{d \lambda}{2 \pi} e^{i \lambda x}\left\langle P S\left|F_{a}^{n \alpha}(0) \bar{\psi}(\lambda n) t^{a} \not h \psi(\lambda n)\right| P S\right\rangle$.
$D_{g}(x)$ is related to the twist-3 quark-gluon correlation function $G_{F}\left(x_{1}, x_{2}\right)$ defined by ${ }^{2}$

$$
\begin{aligned}
\int \frac{d \lambda}{2 \pi} \int \frac{d \mu}{2 \pi} e^{i \lambda x_{1}} e^{i \mu\left(x_{2}-x_{1}\right)}\left\langle P S\left|\bar{\psi}(0) F_{a}^{\alpha n}(\mu n) t^{a} h \psi(\lambda n)\right| P S\right\rangle \\
\quad=M \epsilon^{\alpha p n S_{\perp}} G_{F}\left(x_{1}, x_{2}\right),
\end{aligned}
$$

as

$$
D_{g}(x)=-\int_{-1}^{1} d x_{1} G_{F}\left(x_{1}, x_{1}-x\right)
$$

with the support on $|x|<1$. The relation (15) is also new.

From (7), one can obtain another relation involving $\Delta H_{3 T}(x)$ as follows. We first write

$$
\begin{aligned}
\Phi^{\alpha n, \beta \mu}(x)= & \frac{-i}{x} \int \frac{d \lambda}{2 \pi} \frac{d e^{i \lambda x}}{d \lambda}\left\langle P S\left|F^{\alpha n}(0)[0, \lambda n] F^{\beta \mu}(\lambda n)\right| P S\right\rangle \\
= & \frac{i}{x} \int \frac{d \lambda}{2 \pi} e^{i \lambda x}\langle P S| F^{\alpha n}(0)[0, \lambda n] \\
& \times D^{n}(\lambda n) F^{\beta \mu}(\lambda n)|P S\rangle
\end{aligned}
$$

where we have used the relation $\frac{d}{d \lambda}[0, \lambda n] F^{\beta \mu}(\lambda n)=$ $[0, \lambda n] D^{n}(\lambda n) F^{\beta \mu}(\lambda n)$ after integration by parts. We then use the Bianchi identity $D^{n} F^{\beta \mu}=-D^{\beta} F^{\mu n}+D^{\mu} F^{\beta n}$ to get

$\Phi^{\alpha n, \beta \mu}(x)=\frac{1}{x} \int d x_{1}\left\{N_{D}^{\alpha \beta \mu}\left(x_{1}, x\right)-N_{D}^{\alpha \mu \beta}\left(x_{1}, x\right)\right\}$.

Taking $\alpha, \beta$, and $\mu$ to be transverse, one arrives at the following relation:

$$
x \Delta H_{3 T}(x)=\frac{4}{x} \int d x_{1}\left\{D_{1}\left(x_{1}, x\right)-D_{2}\left(x_{1}, x\right)\right\} .
$$

Using the relations, (11) and (12), in this equation one eventually obtains

$$
\begin{aligned}
\frac{1}{2} x^{2} \Delta H_{3 T}(x)= & 2 \int d x_{1} \mathcal{P} \frac{1}{x-x_{1}}\left\{N\left(x_{1}, x\right)+N\left(x, x-x_{1}\right)\right\} \\
& +\frac{1}{2} \Delta G_{T}^{(1)}(x)
\end{aligned}
$$

This relation was derived here for the first time.

To summarize this subsection, we have obtained two relations (15) and (22) which relate the two intrinsic functions, $\Delta G_{3 T}(x)$ and $\Delta H_{3 T}(x)$, and one kinematical function, $\Delta G_{T}^{(1)}(x)$, to the dynamical functions. One needs another independent relation to express those three functions in terms of the dynamical functions.

\footnotetext{
${ }^{2}$ We follow the convention of $[24,25]$ for $G_{F}\left(x_{1}, x_{2}\right)$.
} 


\section{Constraint relations from nonlocal operator product expansion}

Here we derive a relation from the nonlocal version of the operator product expansion (OPE) for general correlation functions not necessarily on the light cone. The method was originally developed in $[45,64]$, and has been frequently used for the twist-3 distributions $[24,45,63,65,66]$, the twist-3 fragmentation functions $[59,67]$, and the distribution amplitudes for hard exclusive processes $[64,68,69]$, etc. This method is equivalent to OPE and incorporates all the constraints from Lorentz invariance property of the correlation functions. Here we apply this method to the twist-3 gluon distribution functions to derive constraint relations.

We start from the following operator identity:

$$
\begin{aligned}
\frac{\partial}{\partial y_{\rho}}\left[F^{\alpha \nu}(-y)[-y, y] F^{\beta \mu}(y)\right]=- & F^{\alpha \nu}(-y) \overleftarrow{D}^{\rho}(-y)[-y, y] F^{\beta \mu}(y)+F^{\alpha \nu}(-y)[-y, y] \vec{D}^{\rho}(y) F^{\beta \mu}(y) \\
& +i \int_{1}^{-1} d t t F^{\alpha \nu}(-y)[-y, t y] g F^{\rho y}(t y)[t y, y] F^{\beta \mu}(y) .
\end{aligned}
$$

In the left-hand side (l.h.s.) of this equation, one should first make $y$ non-lightlike, and take the light cone limit $y^{\mu} \rightarrow y^{-} g_{-}^{\mu}$ after taking the derivative. From translational invariance, we have another identity,

$$
\begin{aligned}
0= & \lim _{\xi \rightarrow 0} \frac{d}{d \xi_{\rho}}\left\langle P S\left|F^{\alpha \nu}(-y+\xi)[-y+\xi, y+\xi] F^{\beta \mu}(y+\xi)\right| P S\right\rangle \\
= & \left\langle P S\left|F^{\alpha \nu}(-y) \overleftarrow{D}^{\rho}(-y)[-y, y] F^{\beta \mu}(y)\right| P S\right\rangle+\left\langle P S\left|F^{\alpha \nu}(-y)[-y, y] \vec{D}^{\rho}(y) F^{\beta \mu}(y)\right| P S\right\rangle \\
& +i \int_{1}^{-1} d t\left\langle P S\left|F^{\alpha \nu}(-y)[-y, t y] g F^{\rho y}(t y)[t y, y] F^{\beta \mu}(y)\right| P S\right\rangle .
\end{aligned}
$$

We take the expectation value of (23) by $|P S\rangle$, and use (24) to eliminate the first term in the right-hand side (r.h.s.). We then obtain

$$
\begin{aligned}
\frac{\partial}{\partial y_{\rho}}\left\langle P S\left|F^{\alpha \nu}(-y)[-y, y] F^{\beta \mu}(y)\right| P S\right\rangle= & 2\left\langle P S \mid F^{\alpha \nu}(-y)[-y, y] \vec{D}^{\rho}(y) F^{\beta \mu}(y) \| P S\right\rangle \\
& +\left\langle P S\left|i \int_{1}^{-1} d t(t+1) F^{\alpha \nu}(-y)[-y, t y] g F^{\rho y}(t y)[t y, y] F^{\beta \mu}(y)\right| P S\right\rangle .
\end{aligned}
$$

From this equation, one obtains the identity

$$
\begin{aligned}
\frac{\partial}{\partial y^{\beta}}\left\langle P S\left|F^{\alpha y}(-y)[-y, y] F^{\beta y}(y)\right| P S\right\rangle= & \left\langle P S\left|F_{\mu}^{\alpha}(-y)[-y, y] F^{\mu y}(y)\right| P S\right\rangle+2\left\langle P S\left|F^{y \alpha}(-y) g \bar{\psi}(y) y t^{a} \psi(y)\right| P S\right\rangle \\
& +\left\langle P S\left|i \int_{1}^{-1} d t(t+1) F^{\alpha y}(-y)[-y, t y] g F_{\beta}^{y}(t y)[t y, y] F^{\beta y}(y)\right| P S\right\rangle,
\end{aligned}
$$

where we used the QCD e.o.m., $D_{\beta}(y) F^{\beta \mu}(y)=-g \bar{\psi}(y) \gamma^{\mu} t^{a} \psi(y)$, in the second term of the r.h.s. In order to get a relation among the twist-3 distributions from (26), one needs inverse Fourier transform of (1), (3), and (17). In particular, to calculate the 1.h.s. and the first term in the r.h.s. of (26), one has to use the following form:

$$
\begin{aligned}
\left\langle P S\left|F^{\alpha \nu}(-y)[-y, y] F^{\beta \mu}(y)\right| P S\right\rangle= & \int d x e^{-2 i x p \cdot y}\left[\frac{1}{2}\left\{\left(-g_{\perp}^{\alpha \beta} p^{\mu}+g_{\perp}^{\alpha \mu} p^{\beta}\right) p^{\nu}-\left(-g_{\perp}^{\nu \beta} p^{\mu}+g_{\perp}^{\nu \mu} p^{\beta}\right) p^{\alpha}\right\} x G(x)\right. \\
& +\frac{i S \cdot y}{2(p \cdot y)^{2}}\left\{\left(\epsilon^{p y \alpha \beta} p^{\mu}-\epsilon^{p y \alpha \mu} p^{\beta}\right) p^{\nu}-\left(\epsilon^{p y \nu \beta} p^{\mu}-\epsilon^{p y \nu \mu} p^{\beta}\right) p^{\alpha}\right\} x \Delta G(x) \\
& -\frac{i M}{2 p \cdot y}\left\{\left(\epsilon^{\alpha \beta y S_{\perp}} p^{\mu}-\epsilon^{\alpha \mu y S_{\perp}} p^{\beta}\right) p^{\nu}-\left(\epsilon^{\nu \beta y S_{\perp}} p^{\mu}-\epsilon^{\nu \mu y S_{\perp}} p^{\beta}\right) p^{\alpha}\right\} x \Delta G_{3 T}(x) \\
& \left.+\frac{i M}{2 p \cdot y}\left\{\epsilon^{\beta \mu p y}\left(S_{\perp}^{\alpha} p^{\nu}-S_{\perp}^{\nu} p^{\alpha}\right)-\epsilon^{\alpha \mu p y}\left(S_{\perp}^{\beta} p^{\nu}-S_{\perp}^{\nu} p^{\beta}\right)\right\} x \Delta H_{3 T}(x)\right] .
\end{aligned}
$$


In taking the derivative of (27) with respect to $y^{\beta}$, one should use the form $S_{\perp}^{\mu}=S^{\mu}-\frac{S \cdot y}{p \cdot y} p^{\mu}$ and $g_{\perp}^{\mu \nu}=g^{\mu \nu}-\frac{p^{\mu} y^{\nu}+p^{\nu} y^{\mu}}{p \cdot y}$, keep all components of $y^{\mu}$ with $y^{2} \neq 0$, and then take the $y^{\mu} \rightarrow g_{-}^{\mu} y^{-}$limit. With this procedure, we have eventually obtained the following relation:

$$
\begin{aligned}
x \frac{\partial}{\partial x}( & \left.x \Delta G_{3 T}(x)\right)+x \Delta H_{3 T}(x)+x \Delta G(x) \\
= & -2 \frac{d D_{g}(x)}{d x}+4 \int d x_{1} \mathcal{P} \frac{1}{x-x_{1}} \frac{\partial}{\partial x}\left\{2 N\left(x_{1}, x_{1}-x\right)+N\left(x, x-x_{1}\right)-N\left(x_{1}, x\right)\right\} \\
& +4 \int d x_{1} \mathcal{P} \frac{1}{\left(x-x_{1}\right)^{2}}\left\{-N\left(x_{1}, x_{1}-x\right)-2 N\left(x, x-x_{1}\right)+N\left(x_{1}, x\right)\right\} .
\end{aligned}
$$

This relation is independent from (15) and (22), and the three relations (15), (22), and (28) allow one to solve $\Delta G_{3 T}(x), \Delta H_{3 T}(x)$ and $\Delta G_{T}^{(1)}(x)$ in terms of $\Delta G(x)$ and the dynamical functions.

Here we comment on the relations obtained from operator identities other than (23). One can derive a constraint relation by considering the following correlation function:

$y_{\rho}\left[\frac{\partial}{\partial y_{\rho}}\left\langle P S\left|F^{y \nu}(-y)[-y, y] F_{\nu}^{\beta}(y)\right| P S\right\rangle-(\rho \leftrightarrow \beta)\right]$.

We found that this correlator simply gives the relation that is obtained from (15) and (22), which supplies a good consistency check. We also found that the operator identity for the correlator

$y_{\rho}\left[\frac{\partial}{\partial y_{\rho}}\left\langle P S\left|F^{y \nu}(-y)[-y, y] \tilde{F}_{\nu}^{\beta}(y)\right| P S\right\rangle-(\rho \leftrightarrow \beta)\right]$,

with $\tilde{F}^{\beta \nu}=\frac{1}{2} \epsilon^{\beta \nu \rho \tau} F_{\rho \tau}$ gives the same relation as (28), which also serves to confirm our result.

It is interesting to compare our approach and that in [63]. The authors of [63] analyzed the correlator (30) to express $\Delta \hat{G}_{3 T}(x)$ and $\Delta \hat{G}_{T}^{(1)}(x)$ in terms of $\Delta G(x)$ and the dynamical twist-3 distributions. They started from the identity

$$
\begin{aligned}
y_{\rho} & {\left[\frac{\partial}{\partial y_{\rho}}\left\langle P S\left|F^{y \nu}(-y) \tilde{F}_{\nu}^{\beta}(y)\right| P S\right\rangle-(\rho \leftrightarrow \beta)\right] } \\
= & \left\langle P S\left|\left(F^{y \nu}(-y) \tilde{F}^{\beta}{ }_{\nu}(y)-F^{\beta \nu}(-y) \tilde{F}^{y}{ }_{\nu}(y)\right)\right| P S\right\rangle \\
& +y_{\alpha} y_{\rho}\left[\frac{\partial}{\partial y_{\rho}}\left\langle P S\left|F^{\alpha \nu}(-y) \tilde{F}_{\nu}^{\beta}(y)\right| P S\right\rangle-(\rho \leftrightarrow \beta)\right] .
\end{aligned}
$$

The second term in the r.h.s. can be rewritten further to be expressed in terms of the $F$-type functions. In our approach, the 1.h.s. and the first term in the r.h.s. are calculated by using (27) and are expressed in terms of the intrinsic distributions. In this method, $\Delta H_{3 T}(x)$ does not survive in the 1.h.s., while it does appear in the first term of the r.h.s. This procedure leads to the same relation as (28). As for the method of [63], they treated the 1.h.s. of (31) in the same way as ours (although they did not refer to the presence of the $\Delta H_{3 T}$ term). On the other hand, they analyzed the first term in the r.h.s. of (31) in a different way. They did not use the form (27), but rewrote it directly in terms of the $F$-type functions. Therefore they could obtain the constraint relation among the twist-3 distribution functions without recourse to $\Delta H_{3 T}(x)$ contribution at any stage. As we will see in the next subsection, our results for $\Delta G_{3 T}(x)$ and $\Delta G_{T}^{(1)}(x)$ agree with those in [63]. Our approach can also supply the expression for $\Delta H_{3 T}(x)$. (See next subsection.)

\section{Solution for intrinsic and kinematical DFs in terms of twist-2 and dynamical twist-3 DFs}

As we found in previous subsections, Eqs. (15), (22), and (28) constitute a complete set of the independent relations among the twist-3 intrinsic, kinematical, and dynamical DFs. Here we provide a solution for the intrinsic and kinematical functions in terms of the twist-2 and dynamical twist-3 DFs. Taking the sum of (15) and (22), we obtain

$$
\begin{aligned}
x \Delta H_{3 T}(x)= & -x \Delta G_{3 T}(x)-\frac{2}{x} D_{g}(x) \\
& +\frac{8}{x} \int d x_{1} \mathcal{P} \frac{1}{x-x_{1}} \\
& \times\left\{N\left(x, x-x_{1}\right)+N\left(x_{1}, x_{1}-x\right)\right\} .
\end{aligned}
$$

Inserting this into (28) to eliminate $\Delta H_{3 T}(x)$, we have

$$
\begin{aligned}
x^{2} \frac{d}{d x} \Delta G_{3 T}(x)+x \Delta G(x)+2 x \frac{d}{d x}\left(\frac{D_{g}(x)}{x}\right)= & 4 \int d x_{1} \mathcal{P} \frac{1}{x-x_{1}} \frac{\partial}{\partial x}\left\{2 N\left(x_{1}, x_{1}-x\right)+N\left(x, x-x_{1}\right)-N\left(x_{1}, x\right)\right\} \\
& +4 \int d x_{1} \mathcal{P} \frac{1}{\left(x-x_{1}\right)^{2}}\left\{-N\left(x_{1}, x_{1}-x\right)-2 N\left(x, x-x_{1}\right)+N\left(x_{1}, x\right)\right\} \\
& -\frac{8}{x} \int d x_{1} \mathcal{P} \frac{1}{x-x_{1}}\left\{N\left(x, x-x_{1}\right)+N\left(x_{1}, x_{1}-x\right)\right\} .
\end{aligned}
$$


This equation can be integrated to give

$$
\begin{aligned}
\Delta G_{3 T}(x)= & -\int_{\epsilon(x)}^{x} d x_{1} \frac{\Delta G\left(x_{1}\right)}{x_{1}}-2\left\{\frac{D_{g}(x)}{x^{2}}+\int_{\epsilon(x)}^{x} d x_{1} \frac{D_{g}\left(x_{1}\right)}{x_{1}^{3}}\right\} \\
& +\frac{4}{x^{2}} \int_{-1}^{1} d x_{1} \mathcal{P} \frac{1}{x-x_{1}}\left\{2 N\left(x_{1}, x_{1}-x\right)+N\left(x, x-x_{1}\right)-N\left(x_{1}, x\right)\right\} \\
& +\int_{\epsilon(x)}^{x} d x_{2} \frac{8}{x_{2}^{3}} \int_{-1}^{1} d x_{1} \mathcal{P} \frac{1}{x_{2}-x_{1}}\left\{N\left(x_{1}, x_{1}-x_{2}\right)-N\left(x_{1}, x_{2}\right)\right\} \\
& +\int_{\epsilon(x)}^{x} d x_{2} \frac{4}{x_{2}^{2}} \int_{-1}^{1} \mathcal{P} \frac{1}{\left(x_{2}-x_{1}\right)^{2}}\left\{N\left(x_{1}, x_{1}-x_{2}\right)-N\left(x_{2}, x_{2}-x_{1}\right)\right\} .
\end{aligned}
$$

Combining this result and (15), one obtains the expression for $\Delta G_{T}^{(1)}(x)$ as

$$
\begin{aligned}
\Delta G_{T}^{(1)}(x)= & x^{2} \int_{\epsilon(x)}^{x} d x_{1} \frac{\Delta G\left(x_{1}\right)}{x_{1}}+2 x^{2} \int_{\epsilon(x)}^{x} d x_{1} \frac{D_{g}\left(x_{1}\right)}{x_{1}^{3}} \\
& -x^{2} \int_{\epsilon(x)}^{x} d x_{2} \frac{8}{x_{2}^{3}} \int_{-1}^{1} d x_{1} \mathcal{P} \frac{1}{x_{2}-x_{1}}\left\{N\left(x_{1}, x_{1}-x_{2}\right)-N\left(x_{1}, x_{2}\right)\right\} \\
& -x^{2} \int_{\epsilon(x)}^{x} d x_{2} \frac{4}{x_{2}^{2}} \int_{-1}^{1} d x_{1} \mathcal{P} \frac{1}{\left(x_{2}-x_{1}\right)^{2}}\left\{N\left(x_{1}, x_{1}-x_{2}\right)-N\left(x_{2}, x_{2}-x_{1}\right)\right\} .
\end{aligned}
$$

The result in (34) and (35) agrees with that in [63]. Insertion of (34) into (32) gives the expression for $\Delta H_{3 T}(x)$ as

$$
\begin{aligned}
\Delta H_{3 T}(x)= & \int_{\epsilon(x)}^{x} d x_{1} \frac{\Delta G\left(x_{1}\right)}{x_{1}}+2 \int_{\epsilon(x)}^{x} d x_{1} \frac{D_{g}\left(x_{1}\right)}{x_{1}^{3}}+\frac{4}{x^{2}} \int_{-1}^{1} d x_{1} \mathcal{P} \frac{1}{x-x_{1}}\left\{N\left(x, x-x_{1}\right)+N\left(x_{1}, x\right)\right\} \\
& -8 \int_{\epsilon(x)}^{x} d x_{2} \frac{1}{x_{2}^{3}} \int_{-1}^{1} d x_{1} \mathcal{P} \frac{1}{x_{2}-x_{1}}\left\{N\left(x_{1}, x_{1}-x_{2}\right)-N\left(x_{1}, x_{2}\right)\right\} \\
& -4 \int_{\epsilon(x)}^{x} d x_{2} \frac{1}{x_{2}^{2}} \int_{-1}^{1} d x_{1} \mathcal{P} \frac{1}{\left(x_{2}-x_{1}\right)^{2}}\left\{N\left(x_{1}, x_{1}-x_{2}\right)-N\left(x_{2}, x_{2}-x_{1}\right)\right\} .
\end{aligned}
$$

This result is new. As shown in (34), (35), and (36), the intrinsic and kinematical twist-3 gluonic distributions are completely determined by $\Delta G(x)$ (often called Wandzura-Wilczek contribution) and the $F$-type purely gluonic correlation function $N\left(x_{1}, x_{2}\right)$ and the quarkgluon correlation function $G_{F}\left(x_{1}, x_{2}\right)$. Since these relations are model independent exact relations, they need to be satisfied in phenomenological applications. These relations also provide a basis for the renormalization of the intrinsic and the kinematical twist-3 distributions. The evolution equations for $N\left(x_{1}, x_{2}\right)$ and $G_{F}\left(x_{1}, x_{2}\right)$ have already been derived in [55]. The above relations (34), (35), and (36) show it also determines the scale dependence of $\Delta G_{3 T}(x), \Delta G_{T}^{(1)}$ and $\Delta H_{3 T}(x)$.

\section{TWIST-3 GLUON FRAGMENTATION FUNCTIONS}

\section{A. Intrinsic, kinematical, and dynamical twist-3 gluon fragmentation functions}

In this section we extend our analysis in the previous section to the twist-3 gluon fragmentation function. We consider FFs for a spin-1/2 baryon with mass $M_{h}$, four momentum $P_{h}$ $\left(P_{h}^{2}=M_{h}^{2}\right)$, and the spin vector $S\left(S^{2}=-M_{h}^{2}\right)$. In the twist-3 accuracy, we can treat $P_{h}$ as lightlike and introduce another lightlike vector $w$ by the relation $P_{h} \cdot w=1$. We again work in a frame where $P_{h}^{\mu}=P_{h}^{+} g_{+}^{\mu}$ and $w^{\mu}=g_{-}^{\mu} / P_{h}^{+}$. Transverse spin vector for the baryon $S_{\perp}^{\mu}$ is normalized as $S_{\perp}^{2}=-1$. Similarly to (1), the gluon's collinear FFs can be defined from the following fragmentation matrix elements [62]: 


$$
\begin{aligned}
\hat{\Gamma}^{\alpha \nu, \beta \mu}(z)= & \frac{1}{N^{2}-1} \int \frac{d \lambda}{2 \pi} e^{-i \lambda / z} \sum_{X}\left\langle 0\left|\left([\infty w, 0] F^{\alpha \nu}(0)\right)_{a}\right| h X\right\rangle\left\langle h X\left|\left(F^{\beta \mu}(\lambda w)[\lambda w, \infty w]\right)_{a}\right| 0\right\rangle \\
= & \left\{\left(-g_{\perp}^{\alpha \beta} P_{h}^{\mu}+g_{\perp}^{\alpha \mu} P_{h}^{\beta}\right) P_{h}^{\nu}-\left(-g_{\perp}^{\nu \beta} P_{h}^{\mu}+g_{\perp}^{\nu \mu} P_{h}^{\beta}\right) P_{h}^{\alpha}\right\} \hat{G}(z) \\
& +i(S \cdot w)\left\{\left(\epsilon^{P_{h} w \alpha \beta} P_{h}^{\mu}-\epsilon^{P_{h} w \alpha \mu} P_{h}^{\beta}\right) P_{h}^{\nu}-\left(\epsilon^{P_{h} w \nu \beta} P_{h}^{\mu}-\epsilon^{P_{h} w \nu \mu} P_{h}^{\beta}\right) P_{h}^{\alpha}\right\} \Delta \hat{G}(z) \\
& -M_{h}\left[\left\{\left(w^{\alpha} \epsilon^{\beta P_{h} w S_{\perp}}+w^{\beta} \epsilon^{\alpha P_{h} w S_{\perp}}\right) P_{h}^{\mu}-\left(w^{\alpha} \epsilon^{\mu P_{h} w S_{\perp}}+w^{\mu} \epsilon^{\alpha P_{h} w S_{\perp}}\right) P_{h}^{\beta}\right\} P_{h}^{\nu}\right. \\
& \left.-\left\{\left(w^{\nu} \epsilon^{\beta P_{h} w S_{\perp}}+w^{\beta} \epsilon^{\nu P_{h} w S_{\perp}}\right) P_{h}^{\mu}-\left(w^{\nu} \epsilon^{\mu P_{h} w S_{\perp}}+w^{\mu} \epsilon^{\nu P_{h} w S_{\perp}}\right) P_{h}^{\beta}\right\} P_{h}^{\alpha}\right] \Delta \hat{G}_{3 \bar{T}}(z) \\
& -i M_{h}\left\{\left(\epsilon^{\alpha \beta w S_{\perp}} P_{h}^{\mu}-\epsilon^{\alpha \mu w S_{\perp}} P_{h}^{\beta}\right) P_{h}^{\nu}-\left(\epsilon^{\nu \beta w S_{\perp}} P_{h}^{\mu}-\epsilon^{\nu \mu w S_{\perp}} P_{h}^{\beta}\right) P_{h}^{\alpha}\right\} \Delta \hat{G}_{3 T}(z) \\
& +M_{h}\left\{\epsilon^{\beta \mu P_{h} w}\left(S_{\perp}^{\alpha} P_{h}^{\nu}-S_{\perp}^{\nu} P_{h}^{\alpha}\right)+\epsilon^{\alpha \nu P_{h} w}\left(S_{\perp}^{\beta} P_{h}^{\mu}-S_{\perp}^{\mu} P_{h}^{\beta}\right)\right\} \Delta \hat{H}_{3 \bar{T}}(z) \\
& +i M_{h}\left\{\epsilon^{\beta \mu P_{h} w}\left(S_{\perp}^{\alpha} P_{h}^{\nu}-S_{\perp}^{\nu} P_{h}^{\alpha}\right)-\epsilon^{\alpha \nu P_{h} w}\left(S_{\perp}^{\beta} P_{h}^{\mu}-S_{\perp}^{\mu} P_{h}^{\beta}\right)\right\} \Delta \hat{H}_{3 T}(z)+\cdots
\end{aligned}
$$

where $N=3$ is the number colors for $S U(N)$ and $+\cdots$ denotes twist-4 or higher. All functions in (37) are defined as real. Note that the last two terms drop in the correlator $\sim\left\langle F^{w \nu}\right\rangle\left\langle F^{w \mu}\right\rangle$, but we need this general correlator to derive relations among the twist-3 gluonic FFs. $\hat{G}(z)$ and $\Delta \hat{G}(z)$ are, respectively, twist- 2 unpolarized and helicity FFs, and other 4 functions $\Delta \hat{G}_{3 T}(z), \Delta \hat{G}_{3 \bar{T}}(z), \Delta \hat{H}_{3 T}(z)$, and $\Delta \hat{H}_{3 \bar{T}}(z)$ are intrinsic twist-3 FFs. Compared with the distribution functions, the number of twist- 3 FFs is doubled due to the absence of the constraint from time reversal invariance, i.e., naively $T$-odd FFs $\Delta \hat{G}_{3 \bar{T}}(z)$ and $\Delta \hat{H}_{3 \bar{T}}(z)$ survive in addition to "naively $T$-even" $\Delta \hat{G}_{3 T}(z)$ and $\Delta \hat{H}_{3 T}(z)$. Each function in (37) has a support on $0<z<1$.

The second type of a gluon's FFs are the kinematical FFs, which are defined by

$$
\begin{aligned}
\hat{\Gamma}_{\partial}^{\alpha \beta \gamma}(z)= & \frac{1}{N^{2}-1} \sum_{X} \int \frac{d \lambda}{2 \pi} e^{-i \lambda / z}\left\langle 0\left|\left([\infty w, 0] F^{w \alpha}(0)\right)_{a}\right| h X\right\rangle\left\langle h X\left|\left(F^{w \beta}(\lambda w)[\lambda w, \infty w]\right)_{a}\right| 0\right\rangle i \check{\partial}^{\gamma} \\
= & -\frac{M_{h}}{2} g_{\perp}^{\alpha \beta} \epsilon^{\gamma P_{h} w S_{\perp}} \hat{G}_{T}^{(1)}(z)-i \frac{M_{h}}{2} \epsilon^{\alpha \beta P_{h} w} S_{\perp}^{\gamma} \Delta \hat{G}_{T}^{(1)}(z)+\frac{M_{h}}{8}\left(\epsilon^{P_{h} w S_{\perp}\{\alpha} g_{\perp}^{\beta\} \gamma}+\epsilon^{P_{h} w \gamma\{\alpha} S_{\perp}^{\beta\}}\right) \Delta \hat{H}_{T}^{(1)}(z) \\
& +\left(\text { terms proportional to } P_{h}^{\gamma}\right)+\cdots,
\end{aligned}
$$

where $\cdots$ denotes twist-4 or higher. These three kinematical FFs $\hat{G}_{T}^{(1)}(z), \Delta \hat{G}_{T}^{(1)}(z)$, and $\Delta \hat{H}_{T}^{(1)}(z)$ can also be written as the $k_{\perp}^{2} / M_{h}^{2}$-moment of the TMD FFs as in (2) for the distribution functions. Each function has a support on $0<z<1$.

The third type of twist-3 FFs are the dynamical ones which are defined as the three gluon correlation function [37-39]:

$$
\begin{aligned}
\hat{N}_{F}^{\alpha \beta \gamma}\left(\frac{1}{z_{1}}, \frac{1}{z_{2}}\right) & =\frac{i}{N^{2}-1} \sum_{X} \int \frac{d \lambda}{2 \pi} \int \frac{d \mu}{2 \pi} e^{-i \frac{\lambda}{z_{1}}} e^{-i \mu\left(\frac{1}{z_{2}}-\frac{1}{z_{1}}\right)} i f^{a b c}\left\langle 0\left|F_{a}^{w \alpha}(0)\right| h X\right\rangle\left\langle h X\left|F_{b}^{w \beta}(\lambda w) g F_{c}^{w \gamma}(\mu w)\right| 0\right\rangle \\
& =i M_{h}\left[-g_{\perp}^{\alpha \beta} \epsilon^{\gamma P_{h} w S_{\perp}} \hat{N}_{2}\left(\frac{1}{z_{2}}-\frac{1}{z_{1}}, \frac{1}{z_{2}}\right)+g_{\perp}^{\alpha \gamma} \epsilon^{\beta P_{h} w S_{\perp}} \hat{N}_{2}\left(\frac{1}{z_{1}}, \frac{1}{z_{2}}\right)+g_{\perp}^{\beta \gamma} \epsilon^{\alpha P_{h} w S_{\perp}} \hat{N}_{1}\left(\frac{1}{z_{1}}, \frac{1}{z_{2}}\right)\right],
\end{aligned}
$$

where the color indices of the field strength are contracted by the antisymmetric structure constant if ${ }^{a b c}$ and the presence of appropriate gauge links similar to (37) is implied to guarantee gauge invariance of the FFs. There are two independent $F$-type FFs $\hat{N}_{1}\left(\frac{1}{z_{1}}, \frac{1}{z_{2}}\right)$ and $\hat{N}_{2}\left(\frac{1}{z_{1}}, \frac{1}{z_{2}}\right)$ which are in general complex, meaning that the number of independent $F$-type FFs is four times more than the distribution case. $\Re \hat{N}_{1,2}\left(\frac{1}{z_{1}}, \frac{1}{z_{2}}\right)$ are naively $T$-even, while
$\Im \hat{N}_{1,2}\left(\frac{1}{z_{1}}, \frac{1}{z_{2}}\right)$ are naively $T$-odd. $\hat{N}_{1,2}\left(\frac{1}{z_{1}}, \frac{1}{z_{2}}\right)$ have a support on $\frac{1}{z_{2}}>1$ and $\frac{1}{z_{2}}>\frac{1}{z_{1}}>0$. Replacing if ${ }^{a b c}$ by the symmetric structure constants $d^{a b c}$, one can define another $F$-type FFs. Although they appear in a certain cross section, e.g., $p p \rightarrow \Lambda^{\uparrow} X[37,38]$, they are not related to other twist3 FFs. We therefore do not consider those FFs hereafter.

One can also define another set of twist- 3 FFs by the replacement of $g F^{w \gamma}(\mu w) \rightarrow D^{\gamma}(\mu w)$ in (39), which gives 


$$
\begin{aligned}
\hat{N}_{D}^{\alpha \beta \gamma}\left(\frac{1}{z_{1}}, \frac{1}{z_{2}}\right)= & \frac{i}{N^{2}-1} \sum_{X} \int \frac{d \lambda}{2 \pi} \int \frac{d \mu}{2 \pi} e^{-i \frac{\lambda}{z_{1}}} e^{-i \mu\left(\frac{1}{z_{2}}-\frac{1}{z_{1}}\right)}\left\langle 0\left|F_{a}^{w \alpha}(0)\right| h X\right\rangle\left\langle h X\left|\left(F^{w \beta}(\lambda w) \overleftarrow{D}^{\gamma}(\mu w)\right)_{a}\right| 0\right\rangle \\
= & i M_{h}\left[g_{\perp}^{\alpha \beta} \epsilon^{\gamma P_{h} w S_{\perp}} \hat{D}_{2}\left(\frac{1}{z_{1}}, \frac{1}{z_{2}}\right)+g_{\perp}^{\alpha \gamma} \epsilon^{\beta P_{h} w S_{\perp}} \hat{D}_{3}\left(\frac{1}{z_{1}}, \frac{1}{z_{2}}\right)+g_{\perp}^{\beta \gamma} \epsilon^{\alpha P_{h} w S_{\perp}} \hat{D}_{1}\left(\frac{1}{z_{1}}, \frac{1}{z_{2}}\right)\right] \\
& +P_{h}^{\gamma} g_{\perp}^{\alpha \beta} \hat{G}\left(\frac{1}{z_{1}}, \frac{1}{z_{2}}\right) \frac{1}{z_{2}}-i P_{h}^{\gamma} \epsilon^{P_{h} w \alpha \beta}(S \cdot w) \Delta \hat{G}\left(\frac{1}{z_{1}}, \frac{1}{z_{2}}\right) \frac{1}{z_{2}} \\
& +i M_{h} P_{h}^{\gamma} \epsilon^{\alpha \beta w S_{\perp}} \Delta \hat{G}_{3 T}\left(\frac{1}{z_{1}}, \frac{1}{z_{2}}\right) \frac{1}{z_{2}}-M_{h} P_{h}^{\gamma} \epsilon^{P_{h} w S_{\perp}\{\alpha} w^{\beta\}} \Delta \hat{G}_{3 \bar{T}}\left(\frac{1}{z_{1}}, \frac{1}{z_{2}}\right) \frac{1}{z_{2}}
\end{aligned}
$$

where gauge links are suppressed for simplicity. $\hat{D}_{1,2,3}\left(\frac{1}{z_{1}}, \frac{1}{z_{2}}\right)$ are also complex functions, and are called $D$-type FFs. Functions in the last two lines are related to those in (37): From the relation

$$
-z \int d\left(\frac{1}{z_{1}}\right) \hat{N}_{D}^{\alpha \beta \gamma}\left(\frac{1}{z_{1}}, \frac{1}{z}\right) w_{\gamma}=\hat{\Gamma}^{\alpha w, \beta w}(z)
$$

it is easy to see

$$
\int d\left(\frac{1}{z_{1}}\right) \hat{f}\left(\frac{1}{z_{1}}, \frac{1}{z}\right)=\hat{f}(z), \text { for } \hat{f}=\hat{G}, \Delta \hat{G}, \Delta \hat{G}_{3 T}, \Delta \hat{G}_{3 \bar{T}} .
$$

Finally we introduce another dynamical FF defined by

$$
\begin{aligned}
\tilde{\Delta}^{\alpha}\left(\frac{1}{z_{1}}, \frac{1}{z_{2}}\right)= & \frac{1}{N} \sum_{X} \int \frac{d \lambda}{2 \pi} \int \frac{d \mu}{2 \pi} e^{-i \frac{\lambda}{z_{1}}} e^{-i \mu\left(\frac{1}{z_{2}}-\frac{1}{z_{1}}\right)} \\
& \times\left\langle 0\left|F_{a}^{w \alpha}(\mu w)\right| h X\right\rangle\left\langle h X\left|\bar{\psi}_{j}(\lambda w) t^{a} \psi_{i}(0)\right| 0\right\rangle \\
= & M_{h}\left[e^{\alpha P_{h} w S_{\perp}}\left(P_{h}\right)_{i j} \tilde{D}_{F T}\left(\frac{1}{z_{1}}, \frac{1}{z_{2}}\right)\right. \\
& \left.+i S_{\perp}^{\alpha}\left(\gamma_{5} P_{h}\right)_{i j} \tilde{G}_{F T}\left(\frac{1}{z_{1}}, \frac{1}{z_{2}}\right)\right]
\end{aligned}
$$

where the spinor indices $i, j$ are shown explicitly. These two functions $\tilde{D}_{F T}$ and $\tilde{G}_{F T}$ are, in general, complex functions with their naively $T$-even real part and the $T$ odd imaginary part. They have a support on $\frac{1}{z_{1}}>0, \frac{1}{z_{2}}<0$ and $\frac{1}{z_{1}}-\frac{1}{z_{2}}>1$. As we will see below, constraint relations for the twist-3 gluonic FFs involve these $F$-type quarkgluon correlation functions through QCD e.o.m. We collectively call the functions in (39) and (43) dynamical twist-3 FFs.

\section{B. Relations between $D$ - and $F$-type FFs and QCD equation of motion}

The gluon FFs introduced in (37)-(40) are not independent but are related by various operator identities. Using the identity (6), we find $D$-type and $F$-type FFs are related as

$$
\begin{aligned}
\hat{N}_{D}^{\alpha \beta \gamma}\left(\frac{1}{z_{1}}, \frac{1}{z_{2}}\right)= & \mathcal{P}\left(\frac{-1}{\frac{1}{z_{2}}-\frac{1}{z_{1}}}\right) \hat{N}_{F}^{\alpha \beta \gamma}\left(\frac{1}{z_{1}}, \frac{1}{z_{2}}\right) \\
& +\delta\left(\frac{1}{z_{2}}-\frac{1}{z_{1}}\right) \hat{\Gamma}_{\partial}^{\alpha \beta \gamma}\left(z_{1}\right) .
\end{aligned}
$$

An important difference of this relation from the similar one for the distribution function (7) is that the correlator for the kinematical FFs appear directly as the coefficient of the $\delta$-function. This is because $F$-type FFs become 0 at $z_{1}=z_{2}$ due to the support property as shown in [70,71]. From (44), we have

$$
\begin{aligned}
\hat{D}_{1}\left(\frac{1}{z_{1}}, \frac{1}{z_{2}}\right)= & \mathcal{P} \frac{1}{\frac{1}{z_{2}}-\frac{1}{z_{1}}} \hat{N}_{1}\left(\frac{1}{z_{1}}, \frac{1}{z_{2}}\right) \\
& +\delta\left(\frac{1}{z_{1}}-\frac{1}{z_{2}}\right)\left(\frac{-1}{2} \Delta \hat{G}_{T}^{(1)}\left(z_{1}\right)+\frac{i}{4} \Delta \hat{H}_{T}^{(1)}\left(z_{1}\right)\right), \\
\hat{D}_{2}\left(\frac{1}{z_{1}}, \frac{1}{z_{2}}\right)= & \mathcal{P} \frac{-1}{\frac{1}{z_{2}}-\frac{1}{z_{1}}} \hat{N}_{2}\left(\frac{1}{z_{2}}-\frac{1}{z_{1}}, \frac{1}{z_{2}}\right) \\
& +\delta\left(\frac{1}{z_{1}}-\frac{1}{z_{2}}\right)\left(\frac{i}{2} \hat{G}_{T}^{(1)}\left(z_{1}\right)-\frac{i}{4} \Delta \hat{H}_{T}^{(1)}\left(z_{1}\right)\right),
\end{aligned}
$$

$$
\begin{aligned}
\hat{D}_{3}\left(\frac{1}{z_{1}}, \frac{1}{z_{2}}\right)= & \mathcal{P} \frac{1}{\frac{1}{z_{2}}-\frac{1}{z_{1}}} \hat{N}_{2}\left(\frac{1}{z_{1}}, \frac{1}{z_{2}}\right) \\
& +\delta\left(\frac{1}{z_{1}}-\frac{1}{z_{2}}\right)\left(\frac{1}{2} \Delta \hat{G}_{T}^{(1)}\left(z_{1}\right)+\frac{i}{4} \Delta \hat{H}_{T}^{(1)}\left(z_{1}\right)\right) .
\end{aligned}
$$

These relations show $\hat{D}_{1,2,3}\left(\frac{1}{z_{1}}, \frac{1}{z_{2}}\right)$ are completely determined by $\hat{N}_{1,2}\left(\frac{1}{z_{1}}, \frac{1}{z_{2}}\right)$ and the kinematical FFs. Following the same procedure leading to (15) from (7), we can derive the e.o.m. relation by contracting (44) with $g_{\beta \gamma}^{\perp}$ as 


$$
\begin{aligned}
\frac{1}{z}\left(\Delta \hat{G}_{3 \bar{T}}(z)+i \Delta \hat{G}_{3 T}(z)\right)-i \tilde{D}_{F T}(z) \\
=i \int d\left(\frac{1}{z_{1}}\right) P\left(\frac{1}{\frac{1}{z}-\frac{1}{z_{1}}}\right)\left\{-2 \hat{N}_{1}\left(\frac{1}{z_{1}}, \frac{1}{z}\right)\right. \\
\left.\quad-\hat{N}_{2}\left(\frac{1}{z_{1}}, \frac{1}{z}\right)+\hat{N}_{2}\left(\frac{1}{z}-\frac{1}{z_{1}}, \frac{1}{z}\right)\right\} \\
\quad+\frac{1}{2}\left(\hat{G}_{T}^{(1)}(z)+\Delta \hat{H}_{T}^{(1)}(z)\right)+\frac{i}{2} \Delta \hat{G}_{T}^{(1)}(z),
\end{aligned}
$$

where $\tilde{D}_{F T}(z)$ is defined from the dynamical FFs in (43) as

$$
\tilde{D}_{F T}(z) \equiv \frac{2}{C_{F}} \int_{0}^{1 / z} d\left(\frac{1}{z_{1}}\right) \tilde{D}_{F T}\left(\frac{1}{z_{1}}, \frac{1}{z_{1}}-\frac{1}{z}\right),
$$

with $C_{F}=\frac{N^{2}-1}{2 N}$, and it has a support on $0<z<1$. Real and imaginary parts of (48), respectively, read

$$
\begin{aligned}
& \frac{1}{z} \Delta \hat{G}_{3 \bar{T}}(z)+\Im \tilde{D}_{F T}(z) \\
& =\int d\left(\frac{1}{z_{1}}\right) P\left(\frac{1}{\frac{1}{z}-\frac{1}{z_{1}}}\right) \Im\left\{2 \hat{N}_{1}\left(\frac{1}{z_{1}}, \frac{1}{z}\right)\right. \\
& \left.\quad+\hat{N}_{2}\left(\frac{1}{z_{1}}, \frac{1}{z}\right)-\hat{N}_{2}\left(\frac{1}{z}-\frac{1}{z_{1}}, \frac{1}{z}\right)\right\} \\
& \quad+\frac{1}{2}\left(\hat{G}_{T}^{(1)}(z)+\Delta \hat{H}_{T}^{(1)}(z)\right),
\end{aligned}
$$

and

$$
\begin{aligned}
\frac{1}{z} \Delta \hat{G}_{3 T}(z)-\Re \tilde{D}_{F T}(z) \\
=\int d\left(\frac{1}{z_{1}}\right) P\left(\frac{1}{\frac{1}{z}-\frac{1}{z_{1}}}\right) \Re\left\{-2 \hat{N}_{1}\left(\frac{1}{z_{1}}, \frac{1}{z}\right)\right. \\
\left.\quad-\hat{N}_{2}\left(\frac{1}{z_{1}}, \frac{1}{z}\right)+\hat{N}_{2}\left(\frac{1}{z}-\frac{1}{z_{1}}, \frac{1}{z}\right)\right\} \\
\quad+\frac{1}{2} \Delta \hat{G}_{T}^{(1)}(z) .
\end{aligned}
$$

The relation (51) is the FF version of (15).

We can also derive another relation from (44). Following a similar step from (19) to (20), we obtain the following relation:

$$
\begin{aligned}
& \frac{\Delta \hat{H}_{3 \bar{T}}(z)}{z}+i \frac{\Delta \hat{H}_{3 T}(z)}{z} \\
& \quad=i \int d\left(\frac{1}{z_{1}}\right)\left[\hat{D}_{2}\left(\frac{1}{z_{1}}, \frac{1}{z}\right)-\hat{D}_{3}\left(\frac{1}{z_{1}}, \frac{1}{z}\right)\right] .
\end{aligned}
$$

Using (46) and (47) in the r.h.s. of this equation and comparing real and imaginary parts of both sides, one obtains the following two relations:

$$
\begin{aligned}
\frac{\Delta \hat{H}_{3 \bar{T}}(z)}{z}= & \int d\left(\frac{1}{z_{1}}\right) \mathcal{P} \frac{1}{\frac{1}{z}-\frac{1}{z_{1}}} \\
& \times \Im\left[\hat{N}_{2}\left(\frac{1}{z_{1}}, \frac{1}{z}\right)+\hat{N}_{2}\left(\frac{1}{z}-\frac{1}{z_{1}}, \frac{1}{z}\right)\right] \\
& +\frac{1}{2}\left(\Delta \hat{H}_{T}^{(1)}(z)-\hat{G}_{T}^{(1)}(z)\right), \\
\frac{\Delta \hat{H}_{3 T}(z)}{z}= & \int d\left(\frac{1}{z_{1}}\right) \mathcal{P} \frac{-1}{\frac{1}{z}-\frac{1}{z_{1}}} \\
& \times \Re\left[\hat{N}_{2}\left(\frac{1}{z_{1}}, \frac{1}{z}\right)+\hat{N}_{2}\left(\frac{1}{z}-\frac{1}{z_{1}}, \frac{1}{z}\right)\right] \\
& -\frac{1}{2} \Delta \hat{G}_{T}^{(1)}(z) .
\end{aligned}
$$

The second one is the FF version of (22) for the distribution function.

To summarize this section, we have derived two independent relations among the intrinsic, kinematical, and dynamical functions, (51) and (54), for the $T$-even sector, and two independent ones (50) and (53) for the $T$-odd sector. One needs another independent relation for the former and two more relations for the latter.

\section{Constraint relations from nonlocal operator product expansion}

In this subsection, we will derive the relations among the twist-3 gluonic FFs, employing the method used in Sec. II C. To this end, we consider operator identities for the correlation functions away from the light cone which become the fragmentation matrix element in the lightlike limit. We need to calculate a matrix element like

$\frac{\partial}{\partial y_{\rho}}\left\langle 0\left|\left([\infty y,-y] F^{\alpha \nu}(-y)\right)_{a}\right| h X\right\rangle\left\langle h X\left|\left(F^{\beta \mu}(y)[y, \infty y]\right)_{a}\right| 0\right\rangle$,

for $y^{2} \neq 0$ and take the $y^{\mu} \rightarrow \delta_{-}^{\mu} y^{-}$limit after differentiation. To calculate (55), we use the following operator identities:

$$
\begin{aligned}
& \left(F^{\beta \mu}(y)[y, \infty y]\right)_{a} \frac{\overleftarrow{\partial}}{\partial y_{\rho}} \\
& =\left(F^{\beta \mu}(y) \overleftarrow{D}^{\rho}(y)[y, \infty y]\right)_{a} \\
& \quad+i \int_{\infty}^{1} d t t\left(F^{\beta \mu}(y)[y, t y] g F^{\rho y}(t y)[t y, \infty y]\right)_{a}, \\
& \frac{\partial}{\partial y_{\rho}}\left([\infty y,-y] F^{\alpha \nu}(-y)\right)_{a} \\
& =-\left([\infty y,-y] D^{\rho}(-y) F^{\alpha \nu}(-y)\right)_{a} \\
& \quad+i \int_{-1}^{\infty} d t t\left([\infty y, t y] g F^{\rho y}(t y)[t y,-y] F^{\alpha \nu}(-y)\right)_{a} .
\end{aligned}
$$


From translational invariance, we also have the relation

$$
\begin{aligned}
0= & \lim _{\xi \rightarrow 0} \frac{d}{d \xi_{\rho}}\left\langle 0\left|\left([\infty y+\xi,-y+\xi] F^{\alpha \nu}(-y+\xi)\right)_{a}\right| h X\right\rangle\left\langle h X\left|\left(F^{\beta \mu}(y+\xi)[y+\xi, \infty y+\xi]\right)_{a}\right| 0\right\rangle \\
= & \left\langle 0\left|\left([\infty y,-y] F^{\alpha \nu}(-y)\right)_{a}\right| h X\right\rangle\left\langle h X\left|\left(F^{\beta \mu}(y) \overleftarrow{D}^{\rho}(y)[y, \infty y]\right)_{a}\right| 0\right\rangle \\
& +\left\langle 0\left|\left([\infty y,-y] F^{\alpha \nu}(-y)\right)_{a}\right| h X\right\rangle\left\langle h X\left|i \int_{\infty}^{1} d t\left(F^{\beta \mu}(y)[y, t y] g F^{\rho y}(t y)[t y, \infty y]\right)_{a}\right| 0\right\rangle \\
& +\left\langle 0\left|\left([\infty y,-y] D^{\rho}(-y) F^{\alpha \nu}(-y)\right)_{a}\right| h X\right\rangle\left\langle h X\left|\left(F^{\beta \mu}(y)[y, \infty y]\right)_{a}\right| 0\right\rangle \\
& +\left\langle 0\left|i \int_{-1}^{\infty} d t\left([\infty y,-y] g F^{\rho y}(t y)[t y,-y] F^{\alpha \nu}(-y)\right)_{a}\right| h X\right\rangle\left\langle h X\left|\left(F^{\beta \mu}(y)[y, \infty y]\right)_{a}\right| 0\right\rangle .
\end{aligned}
$$

In (55)-(58), we have explicitly written gauge links and color indices. Below we will suppress them for brevity. Inserting (56) and (57) into (55), and using (58) to eliminate the term containing $\left\langle 0\left|D^{\rho}(-y) F^{\alpha \nu}(-y)\right| h X\right\rangle$, one obtains

$$
\begin{aligned}
\frac{\partial}{\partial y_{\rho}}\left\langle 0\left|F^{\alpha \nu}(-y)\right| h X\right\rangle\left\langle h X\left|F^{\beta \mu}(y)\right| 0\right\rangle= & 2\left\langle 0\left|F^{\alpha \nu}(-y)\right| h X\right\rangle\left\langle h X\left|F^{\beta \mu}(y) \overleftarrow{D}^{\rho}(y)\right| 0\right\rangle \\
& +\left\langle 0\left|F^{\alpha \nu}(-y)\right| h X\right\rangle\left\langle h X\left|i \int_{\infty}^{1} d t(t+1) F^{\beta \mu}(y) g F^{\rho y}(t y)\right| 0\right\rangle \\
& +\left\langle 0\left|i \int_{-1}^{\infty} d t(t+1) g F^{\rho y}(t y) F^{\alpha \nu}(-y)\right| h X\right\rangle\left\langle h X\left|F^{\beta \mu}(y)\right| 0\right\rangle .
\end{aligned}
$$

This equation is the starting point of our analysis in this section. Constraint relations for the twist-3 FFs can be obtained by expressing each term of (59) in terms of the FFs defined in Sec. III. 1. To calculate the 1.h.s. of (59), we need the Fourier inversion of $(37)$ for non-lightlike separation $\left(y^{2} \neq 0\right)$, which can be written as

$$
\begin{aligned}
\frac{1}{N^{2}-1} \sum_{X}\left\langle 0\left|F^{\alpha \nu}(-y)\right| h X\right\rangle\left\langle h X\left|F^{\beta \mu}(y)\right| 0\right\rangle= & \int d\left(\frac{1}{z}\right) e^{2 i P_{h} \cdot y / z}\left[\left\{\left(-g_{\perp}^{\alpha \beta} P_{h}^{\mu}+g_{\perp}^{\alpha \mu} P_{h}^{\beta}\right) P_{h}^{\nu}-\left(-g_{\perp}^{\nu \beta} P_{h}^{\mu}+g_{\perp}^{\nu \mu} P_{h}^{\beta}\right) P_{h}^{\alpha}\right\} \hat{G}(z)\right. \\
& +\frac{i(S \cdot y)}{\left(P_{h} \cdot y\right)^{2}}\left\{\left(\epsilon^{P_{h} y \alpha \beta} P_{h}^{\mu}-\epsilon^{P_{h} y \alpha \mu} P_{h}^{\beta}\right) P_{h}^{\nu}-\left(\epsilon^{P_{h} y \nu \beta} P_{h}^{\mu}-\epsilon^{P_{h} y \nu \mu} P_{h}^{\beta}\right) P_{h}^{\alpha}\right\} \Delta \hat{G}(z) \\
& -\frac{M_{h}}{\left(P_{h} \cdot y\right)^{2}}\left[\left\{\left(y^{\alpha} \epsilon^{\beta P_{h} y S_{\perp}}+y^{\beta} \epsilon^{\alpha P_{h} y S_{\perp}}\right) P_{h}^{\mu}-\left(y^{\alpha} \epsilon^{\mu P_{h} y S_{\perp}}+y^{\mu} \epsilon^{\alpha P_{h} y S_{\perp}}\right) P_{h}^{\beta}\right\} P_{h}^{\nu}\right. \\
& \left.-\left\{\left(y^{\nu} \epsilon^{\beta P_{h} y S_{\perp}}+y^{\beta} \epsilon^{\nu P_{h} y S_{\perp}}\right) P_{h}^{\mu}-\left(y^{\nu} \epsilon^{\mu P_{h} y S_{\perp}}+y^{\mu} \epsilon^{\nu P_{h} y S_{\perp}}\right) P_{h}^{\beta}\right\} P_{h}^{\alpha}\right] \Delta \hat{G}_{3 \bar{T}}(z) \\
& -\frac{i M_{h}}{P_{h} \cdot y}\left\{\left(\epsilon^{\alpha \beta y} S_{\perp} P_{h}^{\mu}-\epsilon^{\alpha \mu y S_{\perp}} P_{h}^{\beta}\right) P_{h}^{\nu}-\left(\epsilon^{\nu \beta y S_{\perp}} P_{h}^{\mu}-\epsilon^{\nu \mu y S_{\perp}} P_{h}^{\beta}\right) P_{h}^{\alpha}\right\} \Delta \hat{G}_{3 T}(z) \\
& +\frac{M_{h}}{P_{h} \cdot y}\left\{\epsilon^{\beta \mu P_{h} y}\left(S_{\perp}^{\alpha} P_{h}^{\nu}-S_{\perp}^{\nu} P_{h}^{\alpha}\right)+\epsilon^{\alpha \nu P_{h} y}\left(S_{\perp}^{\beta} P_{h}^{\mu}-S_{\perp}^{\mu} P_{h}^{\beta}\right)\right\} \Delta \hat{H}_{3 \bar{T}}(z) \\
& \left.+\frac{i M_{h}}{P_{h} \cdot y}\left\{\epsilon^{\beta \mu P_{h} y}\left(S_{\perp}^{\alpha} P_{h}^{\nu}-S_{\perp}^{\nu} P_{h}^{\alpha}\right)-\epsilon^{\alpha \nu P_{h} y}\left(S_{\perp}^{\beta} P_{h}^{\mu}-S_{\perp}^{\mu} P_{h}^{\beta}\right)\right\} \Delta \hat{H}_{3 T}(z)+\cdots\right] .
\end{aligned}
$$

In calculating the derivative of the 1.h.s. of (59), one needs to use $S_{\perp}^{\mu}=S^{\mu}-\frac{S \cdot y}{P_{h} \cdot y} P_{h}^{\mu}$ and $g_{\perp}^{\mu \nu}=g^{\mu \nu}-\frac{1}{P_{h} \cdot y}\left(P_{h}^{\mu} y^{\nu}+P_{h}^{\nu} y^{\mu}\right)$ in (60). This way the l.h.s. of (59) can be written in terms of the intrinsic FFs in (60). Likewise the second and the third terms in the r.h.s. of (59) can be easily expressed by using the dynamical FFs in (39). In order to express the first term in the r.h.s. of (59) in terms of the dynamical FFs, we introduce two particular contractions with respect to the Lorentz indices which allows use of the QCD e.o.m. $F_{a}^{\mu \alpha}(y) \overleftarrow{D}_{\mu}(y)=-g \bar{\psi}(y) \gamma^{\alpha} t^{a} \psi(y)$. 


\section{Relations from operator identity I}

We can obtain a constraint relation from the following identity:

$$
\begin{aligned}
y_{\rho}\left[\frac{\partial}{\partial y_{\rho}}\left\langle 0\left|F^{\mu y}(-y)\right| h X\right\rangle\left\langle h X\left|F_{\mu}{ }^{\alpha}(y)\right| 0\right\rangle-(\alpha \leftrightarrow \rho)\right]= & \left\langle 0\left|F^{\mu y}(-y)\right| h X\right\rangle\left\langle h X\left|F_{\mu}{ }^{\alpha}(y)\right| 0\right\rangle-\left\langle 0\left|F^{\mu \alpha}(-y)\right| h X\right\rangle\left\langle h X\left|F_{\mu}{ }^{y}(y)\right| 0\right\rangle \\
& +2\left\langle 0\left|g \bar{\psi}(-y) y t^{a} \psi(-y)\right| h X\right\rangle\left\langle h X\left|F_{a}{ }^{y \alpha}(y)\right| 0\right\rangle \\
& -2 i f^{a b c}\left\langle 0\left|F_{a}{ }^{\mu y}(-y)\right| h X\right\rangle\left\langle h X\left|i g \int_{\infty}^{1} d t F_{b}{ }^{y \alpha}(y) F_{c \mu}{ }^{y}(t y)\right| 0\right\rangle \\
& -i f^{a b c}\left\langle 0\left|F_{a}^{\mu y}(-y)\right| h X\right\rangle\left\langle h X\left|i g \int_{\infty}^{1} d t(t+1) F_{b \mu}{ }^{y}(y) F_{c}{ }^{\alpha y}(t y)\right| 0\right\rangle \\
& -2 i f^{a b c}\left\langle 0\left|i g \int_{-1}^{\infty} d t F_{a}^{\mu y}(t y) F_{b \mu}{ }^{y}(-y)\right| h X\right\rangle\left\langle h X\left|F_{c}{ }^{y \alpha}(y)\right| 0\right\rangle \\
& -i f^{a b c}\left\langle 0\left|i g \int_{-1}^{\infty} d t(t+1) F_{a}^{\alpha y}(t y) F_{b \mu}{ }^{y}(-y)\right| h X\right\rangle\left\langle h X\left|F_{c}^{\mu y}(y)\right| 0\right\rangle .
\end{aligned}
$$

This identity can be obtained as follows: We first use (59) in the 1.h.s. of (61). We then find that the terms corresponding to the first term in the r.h.s. of (59) read

$$
\left\langle 0\left|F^{\mu y}(-y)\right| h X\right\rangle\left\langle h X\left|\left(F_{\mu}{ }^{\alpha}(y) \overleftarrow{D}^{\rho}(y) y_{\rho}-F_{\mu}{ }^{\rho}(y) \overleftarrow{D}^{\alpha}(y) y_{\rho}\right)\right| 0\right\rangle,
$$

which is equal to

$$
-\left\langle 0\left|F^{\mu y}(-y)\right| h X\right\rangle\left\langle h X\left|F^{\alpha \rho}(y) \overleftarrow{D}_{\mu}(y) y_{\rho}\right| 0\right\rangle,
$$

by the Bianchi identity. Then by using the relation (58), it is transformed into

$$
\left\langle 0\left|y_{\beta} D_{\mu}(y) F^{\mu \beta}(-y)\right| h X\right\rangle\left\langle h X\left|F^{\alpha \rho}(y) y_{\rho}\right| 0\right\rangle,
$$

plus terms which contain three field strengths. The former eventually becomes the third term in the r.h.s. of (61) by the QCD e.o.m., and the latter is shown as the fourth and the sixth terms in the r.h.s. of (61).

Using (60) and the inverse Fourier transform of (39) in (61), one obtains the following relation among the twist-3 fragmentation functions:

$$
\begin{aligned}
- & \frac{\partial}{\partial(1 / z)}\left\{\frac{1}{z}\left(\Delta \hat{G}_{3 \bar{T}}(z)-i \Delta \hat{G}_{3 T}(z)\right)\right\}+2 \Delta \hat{G}_{3 \bar{T}}(z)+\frac{\partial}{\partial(1 / z)}\left\{\frac{1}{z}\left(\Delta \hat{H}_{3 \bar{T}}(z)+i \Delta \hat{H}_{3 T}(z)\right)\right\}-2 \Delta \hat{H}_{3 \bar{T}}(z) \\
= & i \frac{\partial}{\partial(1 / z)} \tilde{D}_{F T}^{*}(z)+i \int d\left(\frac{1}{z^{\prime}}\right) \frac{1}{\frac{1}{z}-\frac{1}{z^{\prime}}+i \epsilon} \frac{\partial}{\partial(1 / z)}\left\{-\hat{N}_{2}\left(\frac{1}{z^{\prime}}, \frac{1}{z}\right)-\hat{N}_{2}\left(\frac{1}{z}-\frac{1}{z^{\prime}}, \frac{1}{z}\right)\right\} \\
& +i \int d\left(\frac{1}{z^{\prime}}\right) \frac{1}{\left(\frac{1}{z}-\frac{1}{z^{\prime}}+i \epsilon\right)^{2}}\left\{\hat{N}_{1}\left(\frac{1}{z^{\prime}}, \frac{1}{z}\right)+2 \hat{N}_{2}\left(\frac{1}{z^{\prime}}, \frac{1}{z}\right)-\hat{N}_{2}\left(\frac{1}{z}-\frac{1}{z^{\prime}} \frac{1}{z}\right)\right\} \\
& +i \int d\left(\frac{1}{z^{\prime}}\right) \frac{1}{\frac{1}{z}-\frac{1}{z^{\prime}}-i \epsilon} \frac{\partial}{\partial(1 / z)}\left\{-2 \hat{N}_{1}^{*}\left(\frac{1}{z^{\prime}}, \frac{1}{z}\right)-\hat{N}_{2}^{*}\left(\frac{1}{z^{\prime}}, \frac{1}{z}\right)+\hat{N}_{2}^{*}\left(\frac{1}{z}-\frac{1}{z^{\prime}}, \frac{1}{z}\right)\right\} \\
& +i \int d\left(\frac{1}{z^{\prime}}\right) \frac{1}{\left(\frac{1}{z}-\frac{1}{z^{\prime}}-i \epsilon\right)^{2}}\left\{\hat{N}_{1}^{*}\left(\frac{1}{z^{\prime}}, \frac{1}{z}\right)+\hat{N}_{2}^{*}\left(\frac{1}{z}-\frac{1}{z^{\prime}}, \frac{1}{z}\right)\right\} .
\end{aligned}
$$

The real part of this equation reads 


$$
\begin{aligned}
& -\frac{\partial}{\partial(1 / z)}\left\{\frac{1}{z}\left(\Delta \hat{G}_{3 \bar{T}}(z)-\Delta \hat{H}_{3 \bar{T}}(z)\right)\right\}+2\left(\Delta \hat{G}_{3 \bar{T}}(z)-\Delta \hat{H}_{3 \bar{T}}(z)\right) \\
& =\frac{\partial}{\partial(1 / z)} \mathfrak{\Im} \tilde{D}_{F T}(z)+\int d\left(\frac{1}{z^{\prime}}\right) \frac{1}{\frac{1}{z}-\frac{1}{z^{\prime}}} \frac{\partial}{\partial(1 / z)} \mathfrak{I}\left\{-2 \hat{N}_{1}\left(\frac{1}{z^{\prime}}, \frac{1}{z}\right)+2 \hat{N}_{2}\left(\frac{1}{z}-\frac{1}{z^{\prime}}, \frac{1}{z}\right)\right\} \\
& \quad+\int d\left(\frac{1}{z^{\prime}}\right) \frac{1}{\left(\frac{1}{z}-\frac{1}{z^{\prime}}\right)^{2}} \mathfrak{S}\left\{-2 \hat{N}_{2}\left(\frac{1}{z^{\prime}}, \frac{1}{z}\right)+2 \hat{N}_{2}\left(\frac{1}{z}-\frac{1}{z^{\prime}}, \frac{1}{z}\right)\right\},
\end{aligned}
$$

and the imaginary part gives

$$
\begin{aligned}
\frac{\partial}{\partial(1 / z)}\left\{\frac{1}{z}\left(\Delta \hat{G}_{3 T}(z)+\Delta \hat{H}_{3 T}(z)\right)\right\}= & \frac{\partial}{\partial(1 / z)} \Re \tilde{D}_{F T}(z)+\int d\left(\frac{1}{z^{\prime}}\right) \frac{1}{\frac{1}{z}-\frac{1}{z^{\prime}}} \frac{\partial}{\partial(1 / z)} \mathfrak{R}\left\{-2 \hat{N}_{1}\left(\frac{1}{z^{\prime}}, \frac{1}{z}\right)-2 \hat{N}_{2}\left(\frac{1}{z^{\prime}}, \frac{1}{z}\right)\right\} \\
& +\int d\left(\frac{1}{z^{\prime}}\right) \frac{1}{\left(\frac{1}{z}-\frac{1}{z^{\prime}}\right)^{2}} \Re\left\{2 \hat{N}_{1}\left(\frac{1}{z^{\prime}}, \frac{1}{z}\right)+2 \hat{N}_{2}\left(\frac{1}{z^{\prime}}, \frac{1}{z}\right)\right\}
\end{aligned}
$$

Equations (63) and (64) are the constraint relations among the intrinsic and the dynamical FFs. We note that (64) is the relation obtained as the sum of (51) and (54), while (63) is an independent relation from (50) and (53).

\section{Relations from operator identity II}

Here we use the following identity to get independent relations among the twist-3 FFs:

$$
\begin{aligned}
\frac{\partial}{\partial y^{\mu}}\left\langle 0\left|F^{y \nu}(-y)\right| h X\right\rangle\left\langle h X\left|F^{y \mu}(y)\right| 0\right\rangle= & \left\langle 0\left|F_{\mu}{ }^{\nu}(-y)\right| h X\right\rangle\left\langle 0\left|F^{y \mu}(y)\right| 0\right\rangle+2\left\langle 0\left|F^{y \nu}(-y)\right| h X\right\rangle\left\langle 0\left|g \bar{\psi}(y) \not t^{a} \psi(y)\right| 0\right\rangle \\
& +i f^{a b c}\left\langle 0\left|F_{a}{ }^{y \nu}(-y)\right| h X\right\rangle\left\langle 0\left|i g \int_{\infty}^{1} d t(t+1) F_{b}{ }^{y \rho}(y) F_{c \rho}{ }^{y}(t y)\right| 0\right\rangle \\
& +i f^{a b c}\left\langle 0\left|i g \int_{-1}^{\infty} d t(t+1) F_{a \rho}^{y}(t y) F_{b}{ }^{y \nu}(-y)\right| h X\right\rangle\left\langle 0\left|F_{c}{ }^{y \rho}(t y)\right| 0\right\rangle .
\end{aligned}
$$

This relation is obtained by using (59) in the 1.h.s. of (65), and taking into account of the QCD e.o.m., $D_{\mu}(y) F_{a}^{\mu \alpha}(y)=-g \bar{\psi}(y) \gamma^{\alpha} t^{a} \psi(y)$. For the calculation of the 1.h.s. of this equation, one should use (60) contracted with $y^{\alpha} y^{\beta}$ by keeping $y^{2} \neq 0$ before taking the derivative:

$$
\begin{aligned}
& \frac{1}{N^{2}-} \sum_{X}\left\langle 0\left|F^{y \nu}(-y)\right| h X\right\rangle\left\langle h X\left|F^{y \mu}(y)\right| 0\right\rangle \\
& \quad=\int d\left(\frac{1}{z}\right) e^{2 i P_{h} \cdot y / z}\left[\left\{-g_{\perp}^{\alpha \beta} y_{\alpha} y_{\beta} P_{h}^{\mu} P_{h}^{\nu}+\left(P_{h} \cdot y\right)\left(g_{\perp}^{\mu y} P_{h}^{\nu}+g_{\perp}^{\nu y} P_{h}^{\mu}\right)-g_{\perp}^{\nu \mu}\left(P_{h} \cdot y\right)^{2}\right\} \hat{G}(z)\right. \\
& \quad+i(S \cdot y) \epsilon^{P_{h} y \nu \mu} \Delta \hat{G}(z)-i M_{h} \epsilon^{\nu \mu y S_{\perp}}\left(P_{h} \cdot y\right) \Delta \hat{G}_{3 T}(z) \\
& \left.\quad+M_{h}\left\{\frac{y^{2}}{P_{h} \cdot y}\left(\epsilon^{\mu P_{h} y S_{\perp}} P_{h}^{\nu}+\epsilon^{\nu P_{h} y S_{\perp}} P_{h}^{\mu}\right)-\left(\epsilon^{\mu P_{h} y S_{\perp}} y^{\nu}+\epsilon^{\nu P_{h} y S_{\perp}} y^{\mu}\right)\right\} \Delta \hat{G}_{3 \bar{T}}(z)\right]
\end{aligned}
$$

From the identity (65), one obtains the following relation:

$$
\begin{aligned}
& \frac{\partial}{\partial(1 / z)}\left\{\frac{1}{z} \Delta \hat{G}_{3 \bar{T}}(z)\right\}+\frac{i}{z} \frac{\partial}{\partial(1 / z)} \Delta \hat{G}_{3 T}(z)-3 \Delta \hat{G}_{3 \bar{T}}(z)-\Delta \hat{H}_{3 \bar{T}}(z)+i \Delta \hat{H}_{3 T}(z)+i \Delta \hat{G}(z) \\
& \quad=i \frac{\partial}{\partial(1 / z)} \tilde{D}_{F T}(z)-i \int d\left(\frac{1}{z^{\prime}}\right) \frac{1}{\frac{1}{z}-\frac{1}{z^{\prime}}+i \epsilon} \frac{\partial}{\partial(1 / z)}\left\{2 \hat{N}_{1}\left(\frac{1}{z^{\prime}}, \frac{1}{z}\right)+\hat{N}_{2}\left(\frac{1}{z^{\prime}}, \frac{1}{z}\right)-\hat{N}_{2}\left(\frac{1}{z}-\frac{1}{z^{\prime}}, \frac{1}{z}\right)\right\} \\
& \quad+i \int d\left(\frac{1}{z^{\prime}}\right) \frac{1}{\left(\frac{1}{z}-\frac{1}{z^{\prime}}-i \epsilon\right)^{2}}\left\{\hat{N}_{1}^{*}\left(\frac{1}{z^{\prime}}, \frac{1}{z}\right)+2 \hat{N}_{2}^{*}\left(\frac{1}{z^{\prime}}, \frac{1}{z}\right)-\hat{N}_{2}^{*}\left(\frac{1}{z}-\frac{1}{z^{\prime}}, \frac{1}{z}\right)\right\} .
\end{aligned}
$$

The real part of this equation gives 


$$
\begin{aligned}
& \frac{\partial}{\partial(1 / z)}\left\{\frac{1}{z} \Delta \hat{G}_{3 \bar{T}}(z)\right\}-3 \Delta \hat{G}_{3 \bar{T}}(z)-\Delta \hat{H}_{3 \bar{T}}(z) \\
& =-\frac{\partial}{\partial(1 / z)} \Im \tilde{D}_{F T}(z)+\int d\left(\frac{1}{z^{\prime}}\right) \frac{1}{\frac{1}{z}-\frac{1}{z^{\prime}}} \frac{\partial}{\partial(1 / z)} \mathfrak{s}\left\{2 \hat{N}_{1}\left(\frac{1}{z^{\prime}}, \frac{1}{z}\right)+\hat{N}_{2}\left(\frac{1}{z^{\prime}}, \frac{1}{z}\right)-\hat{N}_{2}\left(\frac{1}{z}-\frac{1}{z^{\prime}}, \frac{1}{z}\right)\right\} \\
& \quad+\int d\left(\frac{1}{z^{\prime}}\right) \frac{1}{\left(\frac{1}{z}-\frac{1}{z^{\prime}}\right)^{2}} \mathfrak{\Im}\left\{\hat{N}_{1}\left(\frac{1}{z^{\prime}}, \frac{1}{z}\right)+2 \hat{N}_{2}\left(\frac{1}{z^{\prime}}, \frac{1}{z}\right)-\hat{N}_{2}\left(\frac{1}{z}-\frac{1}{z^{\prime}}, \frac{1}{z}\right)\right\}
\end{aligned}
$$

while the imaginary part is

$$
\begin{aligned}
& \frac{1}{z} \frac{\partial}{\partial(1 / z)}\left\{\Delta \hat{G}_{3 T}(z)\right\}+\Delta \hat{H}_{3 T}(z)+\Delta \hat{G}(z) \\
& \quad=\frac{\partial}{\partial(1 / z)} \Re \tilde{D}_{F T}(z)-\int d\left(\frac{1}{z^{\prime}}\right) \frac{1}{\frac{1}{z}-\frac{1}{z^{\prime}}} \frac{\partial}{\partial(1 / z)} \mathfrak{R}\left\{2 \hat{N}_{1}\left(\frac{1}{z^{\prime}}, \frac{1}{z}\right)+\hat{N}_{2}\left(\frac{1}{z^{\prime}}, \frac{1}{z}\right)-\hat{N}_{2}\left(\frac{1}{z}-\frac{1}{z^{\prime}}, \frac{1}{z}\right)\right\} \\
& \quad+\int d\left(\frac{1}{z^{\prime}}\right) \frac{1}{\left(\frac{1}{z}-\frac{1}{z^{\prime}}\right)^{2}} \Re\left\{\hat{N}_{1}\left(\frac{1}{z^{\prime}}, \frac{1}{z}\right)+2 \hat{N}_{2}\left(\frac{1}{z^{\prime}}, \frac{1}{z}\right)-\hat{N}_{2}\left(\frac{1}{z}-\frac{1}{z^{\prime}}, \frac{1}{z}\right)\right\} .
\end{aligned}
$$

For consistency check, we have also analyzed the correlation function

$y_{\rho}\left[\frac{\partial}{\partial y_{\rho}}\left\langle 0\left|F^{\mu y}(-y)\right| h X\right\rangle\left\langle h X\left|\tilde{F}_{\mu}{ }^{\alpha}(y)\right| 0\right\rangle-(\alpha \leftrightarrow \rho)\right]$.

This operator only gives the relation among the $T$-even functions which is identical to (69).

To summarize this section, from nonlocal OPE we have derived an independent relation (69) for the $T$-even sector, and two independent relations (63) and (68) for the $T$-odd sector.

\section{Solution for intrinsic and kinematical FFs in terms of dynamical FFs}

Using the constraint relations derived in Secs. III B and III C, we present here expressions for the intrinsic and the kinematical FFs in terms of the twist-2 FFs and the twist-3 dynamical FFs. Since $\hat{N}_{1}\left(\frac{1}{z_{1}}, \frac{1}{z_{2}}\right), \hat{N}_{2}\left(\frac{1}{z_{1}}, \frac{1}{z_{2}}\right)$ and $\hat{N}_{2}\left(\frac{1}{z_{2}}-\frac{1}{z_{1}}, \frac{1}{z_{2}}\right)$ have a support on $\frac{1}{z_{2}}>1$ and $\frac{1}{z_{2}}>\frac{1}{z_{1}}>0$, they vanish at the edge of the support, i.e., $\hat{N}_{1,2}\left(0, \frac{1}{z}\right)=$ $\hat{N}_{1,2}\left(\frac{1}{z}, \frac{1}{z}\right)=\hat{N}_{1,2}\left(\frac{1}{z}, 1\right)=0$. $\tilde{D}_{F T}(z)$ has a support on $z<1$ and thus $\tilde{D}_{F T}(1)=0$. Taking these boundary conditions into account, we can integrate the constraint relations.

\section{T-odd fragmentation functions}

We first integrate (63) to obtain $\Delta \hat{G}_{3 \bar{T}}(z)-\Delta \hat{H}_{3 \bar{T}}(z)$. From that result and (68) one obtains $\Delta \hat{H}_{3 \bar{T}}(z)$ and $\Delta \hat{G}_{3 \bar{T}}(z)$ in terms of the dynamical FFs. Since the calculation is straightforward, we only present the final result. The result for $\Delta \hat{G}_{3 \bar{T}}(z)$ reads

$$
\begin{aligned}
& \Delta \hat{G}_{3 \bar{T}}(z)=-z \mathfrak{s} \tilde{D}_{F T}(z)-\frac{2}{z^{3}} \int_{1}^{1 / z} d\left(\frac{1}{z_{2}}\right) z_{2}^{5} \Im \tilde{D}_{F T}\left(z_{2}\right)-\frac{1}{z} \int_{1}^{1 / z} d\left(\frac{1}{z_{2}}\right) z_{2}^{3} \Im \tilde{D}_{F T}\left(z_{2}\right) \\
& +z \int_{0}^{1 / z} d\left(\frac{1}{z_{1}}\right) \frac{1}{1 / z-1 / z_{1}} \mathfrak{\Im}\left[2 \hat{N}_{1}\left(\frac{1}{z_{1}}, \frac{1}{z}\right)+\hat{N}_{2}\left(\frac{1}{z_{1}}, \frac{1}{z}\right)-\hat{N}_{2}\left(\frac{1}{z}-\frac{1}{z_{1}}, \frac{1}{z}\right)\right] \\
& +\frac{4}{z^{3}} \int_{1}^{1 / z} d\left(\frac{1}{z_{2}}\right) z_{2}^{5} \int_{0}^{1 / z_{2}} d\left(\frac{1}{z_{1}}\right) \frac{1}{1 / z_{2}-1 / z_{1}} \mathfrak{s}\left[\hat{N}_{1}\left(\frac{1}{z_{1}}, \frac{1}{z_{2}}\right)+\hat{N}_{2}\left(\frac{1}{z_{1}}, \frac{1}{z_{2}}\right)\right] \\
& +\frac{2}{z^{3}} \int_{1}^{1 / z} d\left(\frac{1}{z_{2}}\right) z_{2}^{4} \int_{0}^{1 / z_{2}} d\left(\frac{1}{z_{1}}\right) \frac{1}{\left(1 / z_{2}-1 / z_{1}\right)^{2}} \mathfrak{S}\left[\hat{N}_{1}\left(\frac{1}{z_{1}}, \frac{1}{z_{2}}\right)+\hat{N}_{2}\left(\frac{1}{z_{1}}, \frac{1}{z_{2}}\right)\right] \\
& +\frac{2}{z} \int_{1}^{1 / z} d\left(\frac{1}{z_{2}}\right) z_{2}^{3} \int_{0}^{1 / z_{2}} d\left(\frac{1}{z_{1}}\right) \frac{1}{1 / z_{2}-1 / z_{1}} \mathfrak{s}\left[\hat{N}_{1}\left(\frac{1}{z_{1}}, \frac{1}{z_{2}}\right)-\hat{N}_{2}\left(\frac{1}{z_{2}}-\frac{1}{z_{1}}, \frac{1}{z_{2}}\right)\right] \\
& +\frac{1}{z} \int_{1}^{1 / z} d\left(\frac{1}{z_{2}}\right) z_{2}^{2} \int_{0}^{1 / z_{2}} d\left(\frac{1}{z_{1}}\right) \frac{1}{\left(1 / z_{2}-1 / z_{1}\right)^{2}} \Im\left[\hat{N}_{1}\left(\frac{1}{z_{1}}, \frac{1}{z_{2}}\right)+\hat{N}_{2}\left(\frac{1}{z_{1}}, \frac{1}{z_{2}}\right)-2 \hat{N}_{2}\left(\frac{1}{z_{2}}-\frac{1}{z_{1}}, \frac{1}{z_{2}}\right)\right] \text {. }
\end{aligned}
$$


Integrals in this equation can be rewritten as

$$
\int_{1}^{1 / z} d\left(\frac{1}{z_{2}}\right) \int_{0}^{1 / z_{2}} d\left(\frac{1}{z_{1}}\right) \cdots=\int_{z}^{1} \frac{d z_{2}}{z_{2}^{2}} \int_{z_{2}}^{\infty} \frac{d z_{1}}{z_{1}^{2}} \cdots
$$

Similarly the result for $\Delta \hat{H}_{3 \bar{T}}(z)$ is given by

$$
\begin{aligned}
\Delta \hat{H}_{3 \bar{T}}(z)= & -\frac{2}{z^{3}} \int_{1}^{1 / z} d\left(\frac{1}{z_{2}}\right) z_{2}^{5} \Im \tilde{D}_{F T}\left(z_{2}\right)+\frac{1}{z} \int_{1}^{1 / z} d\left(\frac{1}{z_{2}}\right) z_{2}^{3} \Im \tilde{D}_{F T}\left(z_{2}\right) \\
& +z \int_{0}^{1 / z} d\left(\frac{1}{z_{1}}\right) \frac{1}{1 / z-1 / z_{1}} \Im\left[\hat{N}_{2}\left(\frac{1}{z_{1}}, \frac{1}{z}\right)+\hat{N}_{2}\left(\frac{1}{z}-\frac{1}{z_{1}}, \frac{1}{z}\right)\right] \\
& +\frac{4}{z^{3}} \int_{1}^{1 / z} d\left(\frac{1}{z_{2}}\right) z_{2}^{5} \int_{0}^{1 / z_{2}} d\left(\frac{1}{z_{1}}\right) \frac{1}{1 / z_{2}-1 / z_{1}} \Im\left[\hat{N}_{1}\left(\frac{1}{z_{1}}, \frac{1}{z_{2}}\right)+\hat{N}_{2}\left(\frac{1}{z_{1}}, \frac{1}{z_{2}}\right)\right] \\
& +\frac{2}{z^{3}} \int_{1}^{1 / z} d\left(\frac{1}{z_{2}}\right) z_{2}^{4} \int_{0}^{1 / z_{2}} d\left(\frac{1}{z_{1}}\right) \frac{1}{\left(1 / z_{2}-1 / z_{1}\right)^{2}} \Im\left[\hat{N}_{1}\left(\frac{1}{z_{1}}, \frac{1}{z_{2}}\right)+\hat{N}_{2}\left(\frac{1}{z_{1}}, \frac{1}{z_{2}}\right)\right] \\
& -\frac{2}{z} \int_{1}^{1 / z} d\left(\frac{1}{z_{2}}\right) z_{2}^{3} \int_{0}^{1 / z_{2}} d\left(\frac{1}{z_{1}}\right) \frac{1}{1 / z_{2}-1 / z_{1}} \Im\left[\hat{N}_{1}\left(\frac{1}{z_{1}}, \frac{1}{z_{2}}\right)-\hat{N}_{2}\left(\frac{1}{z_{2}}-\frac{1}{z_{1}}, \frac{1}{z_{2}}\right)\right] \\
& -\frac{1}{z} \int_{1}^{1 / z} d\left(\frac{1}{z_{2}}\right) z_{2}^{2} \int_{0}^{1 / z_{2}} d\left(\frac{1}{z_{1}}\right) \frac{1}{\left(1 / z_{2}-1 / z_{1}\right)^{2}} \Im\left[\hat{N}_{1}\left(\frac{1}{z_{1}}, \frac{1}{z_{2}}\right)+\hat{N}_{2}\left(\frac{1}{z_{1}}, \frac{1}{z_{2}}\right)-2 \hat{N}_{2}\left(\frac{1}{z_{2}}-\frac{1}{z_{1}}, \frac{1}{z_{2}}\right)\right] .
\end{aligned}
$$

Using (71) and (73) in (50) and (53), one can obtain the kinematical FFs as

$$
\begin{aligned}
\hat{G}_{T}^{(1)}(z)= & -\frac{2}{z^{2}} \int_{1}^{1 / z} d\left(\frac{1}{z_{2}}\right) z_{2}^{3} \Im \tilde{D}_{F T}\left(z_{2}\right)+\frac{4}{z^{2}} \int_{1}^{1 / z} d\left(\frac{1}{z_{2}}\right) z_{2}^{3} \int_{0}^{1 / z_{2}} d\left(\frac{1}{z_{1}}\right) \frac{1}{1 / z_{2}-1 / z_{1}} \Im\left[\hat{N}_{1}\left(\frac{1}{z_{1}}, \frac{1}{z_{2}}\right)-\hat{N}_{2}\left(\frac{1}{z_{2}}-\frac{1}{z_{1}}, \frac{1}{z_{2}}\right)\right] \\
& +\frac{2}{z^{2}} \int_{1}^{1 / z} d\left(\frac{1}{z_{2}}\right) z_{2}^{2} \int_{0}^{1 / z_{2}} d\left(\frac{1}{z_{1}}\right) \frac{1}{\left(1 / z_{2}-1 / z_{1}\right)^{2}} \Im\left[\hat{N}_{1}\left(\frac{1}{z_{1}}, \frac{1}{z_{2}}\right)+\hat{N}_{2}\left(\frac{1}{z_{1}}, \frac{1}{z_{2}}\right)-2 \hat{N}_{2}\left(\frac{1}{z_{2}}-\frac{1}{z_{1}}, \frac{1}{z_{2}}\right)\right],
\end{aligned}
$$

and

$$
\begin{aligned}
\Delta \hat{H}_{T}^{(1)}(z)= & -\frac{4}{z^{4}} \int_{1}^{1 / z} d\left(\frac{1}{z_{2}}\right) z_{2}^{5} \Im \tilde{D}_{F T}\left(z_{2}\right)+\frac{8}{z^{4}} \int_{1}^{1 / z} d\left(\frac{1}{z_{2}}\right) z_{2}^{5} \int_{0}^{1 / z_{2}} d\left(\frac{1}{z_{1}}\right) \frac{1}{1 / z_{2}-1 / z_{1}} \Im\left[\hat{N}_{1}\left(\frac{1}{z_{1}}, \frac{1}{z_{2}}\right)+\hat{N}_{2}\left(\frac{1}{z_{1}}, \frac{1}{z_{2}}\right)\right] \\
& +\frac{4}{z^{4}} \int_{1}^{1 / z} d\left(\frac{1}{z_{2}}\right) z_{2}^{4} \int_{0}^{1 / z_{2}} d\left(\frac{1}{z_{1}}\right) \frac{1}{\left(1 / z_{2}-1 / z_{1}\right)^{2}} \Im\left[\hat{N}_{1}\left(\frac{1}{z_{1}}, \frac{1}{z_{2}}\right)+\hat{N}_{2}\left(\frac{1}{z_{1}}, \frac{1}{z_{2}}\right)\right] .
\end{aligned}
$$

\section{T-even fragmentation function}

The solution for $\Delta \hat{G}_{3 T}(z), \Delta \hat{G}_{T}^{(1)}(z)$ and $\Delta \hat{H}_{3 T}(z)$ can be obtained by integrating the relations (51), (54), and (69). Actually we can make a shortcut. Since they are in parallel with (15), (22), and (28) for the gluon distributions $\Delta G_{3 T}(x)$, $\Delta G_{T}^{(1)}(x)$, and $\Delta H_{3 T}(x)$, we can read off the desired results from (34), (35), and (36) by a simple replacement. The results read

$$
\begin{aligned}
\Delta \hat{G}_{3 T}(z)= & -\frac{1}{z} \int_{1}^{1 / z} d\left(\frac{1}{z_{2}}\right) z_{2}^{2} \Delta \hat{G}\left(z_{2}\right)+z \Re \tilde{D}_{F T}(z)+\frac{1}{z} \int_{1}^{1 / z} d\left(\frac{1}{z_{2}}\right) z_{2}^{3} \Re \tilde{D}_{F T}\left(z_{2}\right) \\
& -z \int_{0}^{1 / z} d\left(\frac{1}{z_{1}}\right) \frac{1}{\frac{1}{z}-\frac{1}{z_{1}}} \Re\left[2 \hat{N}_{1}\left(\frac{1}{z_{1}}, \frac{1}{z}\right)+\hat{N}_{2}\left(\frac{1}{z_{1}}, \frac{1}{z}\right)-\hat{N}_{2}\left(\frac{1}{z}-\frac{1}{z_{1}}, \frac{1}{z}\right)\right] \\
& -\frac{2}{z} \int_{1}^{1 / z} d\left(\frac{1}{z_{2}}\right) z_{2}^{3} \int_{0}^{1 / z_{2}} d\left(\frac{1}{z_{1}}\right) \frac{1}{\frac{1}{z_{2}}-\frac{1}{z_{1}}} \Re\left[\hat{N}_{1}\left(\frac{1}{z_{1}}, \frac{1}{z_{2}}\right)-\hat{N}_{2}\left(\frac{1}{z_{2}}-\frac{1}{z_{1}}, \frac{1}{z_{2}}\right)\right] \\
& -\frac{1}{z} \int_{1}^{1 / z} d\left(\frac{1}{z_{2}}\right) z_{2}^{2} \int_{0}^{1 / z_{2}} d\left(\frac{1}{z_{1}}\right) \frac{1}{\left(\frac{1}{z_{2}}-\frac{1}{z_{1}}\right)^{2}} \Re\left[\hat{N}_{1}\left(\frac{1}{z_{1}}, \frac{1}{z_{2}}\right)-\hat{N}_{2}\left(\frac{1}{z_{1}}, \frac{1}{z_{2}}\right)\right],
\end{aligned}
$$




$$
\begin{aligned}
\Delta \hat{G}_{T}^{(1)}(z)= & \frac{1}{z^{2}} \int_{1}^{1 / z} d\left(\frac{1}{z_{2}}\right) z_{2}^{2} \Delta \hat{G}\left(z_{2}\right)-\frac{2}{z^{2}} \int_{1}^{1 / z} d\left(\frac{1}{z_{2}}\right) z_{2}^{3} \Re \tilde{D}_{F T}\left(z_{2}\right) \\
& +\frac{4}{z^{2}} \int_{1}^{1 / z} d\left(\frac{1}{z_{2}}\right) z_{2}^{3} \int_{0}^{1 / z_{2}} d\left(\frac{1}{z_{1}}\right) \frac{1}{\frac{1}{z_{2}}-\frac{1}{z_{1}}} \Re\left[\hat{N}_{1}\left(\frac{1}{z_{1}}, \frac{1}{z_{2}}\right)-\hat{N}_{2}\left(\frac{1}{z_{2}}-\frac{1}{z_{1}}, \frac{1}{z_{2}}\right)\right] \\
& +\frac{2}{z^{2}} \int_{1}^{1 / z} d\left(\frac{1}{z_{2}}\right) z_{2}^{2} \int_{0}^{1 / z_{2}} d\left(\frac{1}{z_{1}}\right) \frac{1}{\left(\frac{1}{z_{2}}-\frac{1}{z_{1}}\right)^{2}} \Re\left[\hat{N}_{1}\left(\frac{1}{z_{1}}, \frac{1}{z_{2}}\right)-\hat{N}_{2}\left(\frac{1}{z_{1}}, \frac{1}{z_{2}}\right)\right],
\end{aligned}
$$

and

$$
\begin{aligned}
\Delta \hat{H}_{3 T}(z)= & \frac{1}{z} \int_{1}^{1 / z} d\left(\frac{1}{z_{2}}\right) z_{2}^{2} \Delta \hat{G}\left(z_{2}\right)-\frac{1}{z} \int_{1}^{1 / z} d\left(\frac{1}{z_{2}}\right) z_{2}^{3} \Re \tilde{D}_{F T}\left(z_{2}\right)-z \int_{0}^{1 / z} d\left(\frac{1}{z_{1}}\right) \frac{1}{\frac{1}{z}-\frac{1}{z_{1}}} \Re\left[\hat{N}_{2}\left(\frac{1}{z_{1}}, \frac{1}{z}\right)+\hat{N}_{2}\left(\frac{1}{z}-\frac{1}{z_{1}}, \frac{1}{z}\right)\right] \\
& +\frac{2}{z} \int_{1}^{1 / z} d\left(\frac{1}{z_{2}}\right) z_{2}^{3} \int_{0}^{1 / z_{2}} d\left(\frac{1}{z_{1}}\right) \frac{1}{\frac{1}{z_{2}}-\frac{1}{z_{1}}} \Re\left[\hat{N}_{1}\left(\frac{1}{z_{1}}, \frac{1}{z_{2}}\right)-\hat{N}_{2}\left(\frac{1}{z_{2}}-\frac{1}{z_{1}}, \frac{1}{z_{2}}\right)\right] \\
& +\frac{1}{z} \int_{1}^{1 / z} d\left(\frac{1}{z_{2}}\right) z_{2}^{2} \int_{0}^{1 / z_{2}} d\left(\frac{1}{z_{1}}\right) \frac{1}{\left(\frac{1}{z_{2}}-\frac{1}{z_{1}}\right)^{2}} \Re\left[\hat{N}_{1}\left(\frac{1}{z_{1}}, \frac{1}{z_{2}}\right)-\hat{N}_{2}\left(\frac{1}{z_{1}}, \frac{1}{z_{2}}\right)\right] .
\end{aligned}
$$

This completes the derivation of all the relations among the twist-3 gluonic FFs.

To summarize this section, we have derived all the constraint relations for twist-3 gluonic FFs, which follow from the QCD e.o.m. and the operator product expansion. These relations are exact and need to be taken into account in deriving a twist- 3 cross section to which they contribute, and should constitute a cornerstone for proving the gauge invariance and Lorentz invariance of the cross sections. In particular, the intrinsic and kinematical twist-3 FFs are completely determined by the twist- $2 \mathrm{FF}$ and the dynamical twist-3 FFs (which equal three-gluon correlation functions), which provides a basis for the renormalization of the intrinsic and kinematical FFs.

\section{SUMMARY}

In this paper, we have performed a systematic study on the collinear twist-3 gluonic distribution functions (DFs) and fragmentation functions (FFs). Both DFs and FFs are classified into three categories, intrinsic, kinematical, and dynamical functions. Although they are convenient tools to describe twist 3 cross sections, they are not independent of each other but are constrained by a set of exact relations which follow from the QCD e.o.m. and the nonlocal operator product expansion. We have derived all those constraint relations for all the gluonic twist-3 DFs and FFs and have given expressions for the intrinsic and kinematical DFs and FFs in terms of the dynamical ones. Those relations are expected to play a critical role to guarantee gauge invariance and the Lorentz invariance of the twist-3 cross sections to which those DFs and FFs contribute. Those relations need to be satisfied for phenomenological analyses.

\section{ACKNOWLEDGMENTS}

This work has been supported by the Grant-in-Aid for Scientific Research from the Japanese Society of Promotion of Science under Contracts No. 19K03843 (Y. K.) and No. $18 \mathrm{~J} 11148$ (K. Y.), National Natural Science Foundation of China under Project No. 11435004 and research startup funding at South China Normal University.
[1] R. L. Jaffe, Comments Nucl. Part. Phys. 19, 239 (1990).

[2] A. V. Belitsky, X. D. Ji, W. Lu, and J. Osborne, Phys. Rev. D 63, 094012 (2001).

[3] J. W. Qiu and G. F. Sterman, Nucl. Phys. B378, 52 (1992).
[4] J.-W. Qiu and G. F. Sterman, Phys. Rev. D 59, 014004 (1998).

[5] Y. Kanazawa and Y. Koike, Phys. Lett. B 478, 121 (2000); 490, 99 (2000). 
[6] X. Ji, J. W. Qiu, W. Vogelsang, and F. Yuan, Phys. Rev. D 73, 094017 (2006).

[7] C. Kouvaris, J. W. Qiu, W. Vogelsang, and F. Yuan, Phys. Rev. D 74, 114013 (2006).

[8] Y. Koike and K. Tanaka, Phys. Rev. D 76, 011502(R) (2007).

[9] Y. Koike and T. Tomita, Phys. Lett. B 675, 181 (2009).

[10] W. Vogelsang and F. Yuan, Phys. Rev. D 79, 094010 (2009).

[11] Y. Koike and S. Yoshida, Phys. Rev. D 84, 014026 (2011).

[12] Y. Koike and S. Yoshida, Phys. Rev. D 85, 034030 (2012).

[13] Z. B. Kang, F. Yuan, and J. Zhou, Phys. Lett. B 691, 243 (2010).

[14] K. Kanazawa and Y. Koike, Phys. Lett. B 701, 576 (2011).

[15] A. Metz and D. Pitonyak, Phys. Lett. B 723, 365 (2013); 762, 549(E) (2016).

[16] H. Beppu, K. Kanazawa, Y. Koike, and S. Yoshida, Phys. Rev. D 89, 034029 (2014).

[17] K. Kanazawa, Y. Koike, A. Metz, and D. Pitonyak, Phys. Rev. D 91, 014013 (2015).

[18] Z. B. Kang and F. Yuan, Phys. Rev. D 84, 034019 (2011).

[19] Y. Hatta, B. W. Xiao, S. Yoshida, and F. Yuan, Phys. Rev. D 94, 054013 (2016).

[20] Y. Hatta, B. W. Xiao, S. Yoshida, and F. Yuan, Phys. Rev. D 95, 014008 (2017).

[21] S. Benić and Y. Hatta, Phys. Rev. D 98, 094025 (2018).

[22] S. Benić and Y. Hatta, Phys. Rev. D 99, 094012 (2019).

[23] X. Ji, J. W. Qiu, W. Vogelsang, and F. Yuan, Phys. Lett. B 638, 178 (2006).

[24] H. Eguchi, Y. Koike, and K. Tanaka, Nucl. Phys. B752 (2006).

[25] H. Eguchi, Y. Koike, and K. Tanaka, Nucl. Phys. B763, 198 (2007).

[26] F. Yuan and J. Zhou, Phys. Rev. Lett. 103, 052001 (2009).

[27] H. Beppu, Y. Koike, K. Tanaka, and S. Yoshida, Phys. Rev. D 82, 054005 (2010).

[28] Y. Koike, K. Tanaka, and S. Yoshida, Phys. Rev. D 83, 114014 (2011).

[29] K. Kanazawa and Y. Koike, Phys. Rev. D 88, 074022 (2013).

[30] S. Yoshida, Phys. Rev. D 93, 054048 (2016).

[31] H. Xing and S. Yoshida, Phys. Rev. D 100, 054024 (2019).

[32] S. Benić, Y. Hatta, H. N. Li, and D. J. Yang, Phys. Rev. D 100, 094027 (2019).

[33] Y. Kanazawa and Y. Koike, Phys. Rev. D 64, 034019 (2001).

[34] J. Zhou, F. Yuan, and Z.-T. Liang, Phys. Rev. D 78, 114008 (2008).

[35] Y. Koike, K. Yabe, and S. Yoshida, Phys. Rev. D 92, 094011 (2015).

[36] Y. Koike, A. Metz, D. Pitonyak, K. Yabe, and S. Yoshida, Phys. Rev. D 95, 114013 (2017).

[37] K. Yabe, Y. Koike, A. Metz, D. Pitonyak, and S. Yoshida, J. Phys. Soc. Jpn. Conf. Proc. 26, 021016 (2019).

[38] K. Yabe, Y. Koike, A. Metz, D. Pitonyak, and S. Yoshida, Proc. Sci., SPIN2018 (2019) 192.
[39] L. Gamberg, Z. B. Kang, D. Pitonyak, M. Schlegel, and S. Yoshida, J. High Energy Phys. 01 (2019) 111.

[40] R. L. Jaffe and X. D. Ji, Nucl. Phys. B375, 527 (1992).

[41] Z. T. Liang, A. Metz, D. Pitonyak, A. Schäfer, Y. K. Song, and J. Zhou, Phys. Lett. B 712, 235 (2012).

[42] Y. Hatta, K. Kanazawa, and S. Yoshida, Phys. Rev. D 88, 014037 (2013).

[43] Y. Koike, D. Pitonyak, Y. Takagi, and S. Yoshida, Phys. Lett. B 752, 95 (2016).

[44] Y. Koike, D. Pitonyak, and S. Yoshida, Phys. Lett. B 759, 75 (2016).

[45] I. I. Balitsky and V. M. Braun, Nucl. Phys. B311, 541 (1989).

[46] A. Ali, V. M. Braun, and G. Hiller, Phys. Lett. B 266, 117 (1991).

[47] Y. Koike and K. Tanaka, Phys. Rev. D 51, 6125 (1995).

[48] J. Kodaira, Y. Yasui, K. Tanaka, and T. Uematsu, Phys. Lett. B 387, 855 (1996).

[49] I. I. Balitsky, V. M. Braun, Y. Koike, and K. Tanaka, Phys. Rev. Lett. 77, 3078 (1996).

[50] Y. Koike and N. Nishiyama, Phys. Rev. D 55, 3068 (1997).

[51] A. V. Belitsky and D. Mueller, Nucl. Phys. B503, 279 (1997).

[52] A. V. Belitsky, Phys. Lett. B 405, 312 (1997).

[53] Z. B. Kang and J. W. Qiu, Phys. Rev. D 79, 016003 (2009).

[54] J. Zhou, F. Yuan, and Z. T. Liang, Phys. Rev. D 79, 114022 (2009).

[55] V. M. Braun, A. N. Manashov, and B. Pirnay, Phys. Rev. D 80, 114002 (2009); 86, 119902 (2012).

[56] Z. B. Kang, Phys. Rev. D 83, 036006 (2011).

[57] J. P. Ma and H. Z. Sang, J. High Energy Phys. 04 (2011) 062.

[58] J. Zhou, Phys. Rev. D 92, 074016 (2015).

[59] K. Kanazawa, Y. Koike, A. Metz, D. Pitonyak, and M. Schlegel, Phys. Rev. D 93, 054024 (2016).

[60] K. Kanazawa, A. Metz, D. Pitonyak, and M. Schlegel, Phys. Lett. B 744, 385 (2015).

[61] X. D. Ji, Phys. Lett. B 289, 137 (1992).

[62] P. J. Mulders and J. Rodrigues, Phys. Rev. D 63, 094021 (2001).

[63] Y. Hatta, K. Tanaka, and S. Yoshida, J. High Energy Phys. 02 (2013) 003.

[64] I. I. Balitsky, V. M. Braun, and A. V. Kolesnichenko, Nucl. Phys. B312, 509 (1989).

[65] J. Kodaira and K. Tanaka, Prog. Theor. Phys. 101, 191 (1999).

[66] V. M. Braun, G. P. Korchemsky, and A. N. Manashov, Nucl. Phys. B603, 69 (2001).

[67] I. I. Balitsky and V. M. Braun, Nucl. Phys. B361, 93 (1991).

[68] V. M. Braun and I. E. Filyanov, Z. Phys. C 48, 239 (1990).

[69] P. Ball, V. M. Braun, Y. Koike, and K. Tanaka, Nucl. Phys. B529, 323 (1998).

[70] S. Meissner and A. Metz, Phys. Rev. Lett. 102, 172003 (2009).

[71] L. P. Gamberg, A. Mukherjee, and P. J. Mulders, Phys. Rev. D 77, 114026 (2008). 